\title{
Rabbit Population Landscape-Scale Simulation to Investigate the Relevance of Using Rabbits in Regulatory Environmental Risk Assessment
}

\author{
Christopher J. Topping ${ }^{1}$ - Gabriel S. Weyman ${ }^{2}$
}

Received: 15 December 2016 / Accepted: 9 October 2017 /Published online: 10 November 2017

(C) The Author(s) 2017. This article is an open access publication

\begin{abstract}
This paper describes the development and testing of the ALMaSS rabbit model and its baseline, and subsequently its application to the question of lagomorph population vulnerability in environmental risk assessment (ERA). Development and testing following a pattern-oriented modelling protocol resulted in a model able to replicate local and landscape-level rabbit population patterns. We then tested how robust rabbit populations are to an (imaginary) extreme toxic stressor at a landscape level in a variety of landscapes, and to what extremes key uncertain model parameters must be pushed to cause extinctions. This was contrasted with the same (imaginary) toxic stressor applied to the already existing ALMaSS hare model. For EU risk assessment of plant protection products, these results clearly indicate that if the protection goal is population-level impacts, either in abundance and/ or distribution, then the hare is a much more vulnerable species than the rabbit under all the conditions tested. Rabbits would only be more vulnerable than hares if the entire population were to be exposed simultaneously, when lower body mass would then be a critical factor. This did not occur even though the toxicant and exposure scenarios tested here were extreme and, in fragmented landscapes at scales used here, will not occur in reality from the use of plant protection products on crop fields. As well as specifically answering the question on rabbit versus hare vulnerability, this study
\end{abstract}

Christopher J. Topping

cjt@bios.au.dk

1 Department of Bioscience-Biodiversity and Conservation, Aarhus University, Grenåvej 14, DK-8410 Rønde, Denmark

2 Adama Agricultural Solutions UK Ltd, Unit 15, Thatcham Business Village, Colthrop Way, Thatcham, Berkshire RG19 4LW, UK generally illustrates the potential application of models for setting focal species for risk assessments.

Keywords Oryctolagus cuniculus $\cdot$ European hare $\cdot$ Lepus europaeus $\cdot$ ALMaSS $\cdot$ Agent-based model $\cdot$ Landscape context

\section{Introduction}

Rabbits and hares have become increasingly important in regulatory environmental risk assessment (ERA) of plant protection products (PPPs) in the EU in recent years. According to the relevant guidance in 2002 [1], leafy crops scenario risk assessment included a 3000-g hare, but no other crop scenario included a lagomorph (orchards/vines/hops/cereals/grassland - no lagomorph). However, according to the latest guidance [2], rabbits and hares are relevant in many more crop scenarios:

Rabbit (1543 g) - cereals, leafy vegetables, legume forage, oilseed rape, orchards, potatoes, pulses, strawberries, sugar beet and sunflower.

Hare (3800 g)-grassland, vineyard.

Traditionally, risk assessment for mammals only considered the individual, and thus lagomorphs are not necessarily the worst-case species in the regulatory risk assessment, which tends to be small mammals such as mouse and vole due to their lower body weight and higher metabolism resulting in higher food intake rate relative to bodyweight [2]. As food eaten in a treated field is assumed to be contaminated with the plant protection product, this results in higher relative intake of the toxicant by the smaller mammals. However, lack of experience and a surprising lack of relevant published data to 
refine the basic and conservative initial risk assessment have resulted in lagomorphs being more of a challenge in the risk assessment than previously. In addition, new directions in European risk assessment see a change towards population and landscape-level risk assessment and inclusion of system characteristics [3, 4], which will almost certainly be extended to mammals in future guidance.

In this paper, when referring to rabbit, we mean European Rabbit (Oryctolagus cuniculus), and when referring to hare, we mean European Brown Hare (Lepus europaeus). Two other species of hare can be relevant in the EU arable landscape (Iberian Hare (Lepus granatensis) and Mountain Hare (Lepus timidus (hibernicus)), but have not been explicitly modelled here.

Both rabbits and hares are present in many crop scenarios, but the smaller rabbit is taken as protective of the larger hare, so is used in the protective risk assessment. However, while rabbit may be more sensitive than hare, due to its lower bodyweight, the question we pose here is, is it more ecologically vulnerable? Both species are hunted, and rabbits are often considered a pest [e.g. 5-8]. Therefore, assuming a lagomorph is used in risk assessment, should the focal species be the rabbit, or hare?

We use an ecological model to investigate the relative ecological vulnerability of each species, which can help risk managers make decisions concerning relevance and acceptability of the risk assessment for each species. A highly detailed hare model already used for risk assessment was available in the ALMaSS system [9, 10], but a rabbit model needed to be developed. In developing this model, the critical step is development of a population baseline against which changes poststressor application can be compared.

This paper describes the development of the rabbit model and its baseline, and subsequently its application to the question of lagomorph population vulnerability in ERA. We tested how robust rabbit populations are to an (imaginary) extreme toxic stressor at a landscape level in a variety of landscapes, and to what extremes key uncertain model parameters must be pushed to cause extinctions. This was contrasted with the same (imaginary) toxic stressor applied to the ALMaSS hare model. The results are then discussed in terms of relevance of either species as a focal species for risk assessment of plant protection products in the EU. Uncertainties and gaps in current scientific knowledge that caused difficulties in parameterizing the rabbit model are also discussed.

\section{Methods}

The methods are separated into two distinct sections. The first covers model development, the second the methods used to evaluate lagomorph population responses to pesticide stressors.

\subsection{Rabbit Model Development}

The rabbit model was developed using pattern-oriented modelling (POM), a commonly used method for developing agent-based models [e.g. 11-15]. POM refers to the multicriteria design, selection and calibration of models of complex systems [16]. The approach is to use observed patterns, which are characteristic of a certain system, for detecting the mechanisms that generate these patterns and therefore are likely to be key elements of the system's internal organisation [17]. Multiple patterns are used, which are observed at different scales and hierarchical levels to characterise complex systems and their dynamics.

POM is comprised of three interrelated elements. First, multiple patterns should be used for model design, i.e. a model should not only include those factors which are considered essential for the model's purpose, but also entities and processes that would allow patterns to emerge which are considered characteristic for the system's structure and functioning [18]. Criteria are defined for deciding whether the model reproduces each pattern. The model is then revised until the most important patterns observed in reality also emerge in the model. This model revision may include the second element, which is the contrasting of alternative sub-models representing a certain key process. For this, the alternative sub-models, for example of foraging, competition or habitat selection, are implemented one at a time in the full model. Then, the alternatives are tested by testing how well the full model reproduces the set of characteristic patterns defined before. Sub-models that cannot reproduce one or more patterns are rejected. This is repeated until the best sub-model has been identified. Thirdly, multiple patterns are used for calibrating the model parameters. Similar to sub-model selection, each pattern is used as a criterion for acceptance for parameter values. This approach is similar to 'inverse modelling' or 'Monte Carlo filtering' techniques used in other disciplines [19].

Here we use the term 'signal pattern' to represent realworld observations against which we attempt to match 'output patterns' from the model.

\subsubsection{Parameter Fitting}

Once cyclic implementation and testing of sub-model components produced output patterns that appeared to respond similarly to the real world, then a parameter fitting exercise was carried out. Parameter fitting used a guided fitting approach [14] whereby an overall fit statistic was calculated based on the sum of squared deviance of model produced output patterns compared to real world input pattern values. In this case, no weights were applied and all patterns contributed equally to the overall fit. 
A cyclic approach was used to generate the selected best-fit parameter value set:

1) Parameter inputs to rabbit model mechanisms were identified and a range of parameter values used for all values to create a small one-at-a-time sensitivity analysis. The range was $\pm 10,20,50 \%$, or the nearest integer values in cases where the parameter was not a real number. Bestfit points were found from this data set.

2) Analysis of response curves was used to estimate new better fitting values. If better fitting values could be found, then estimation continued by trial-and-error until no significant progress could be made by hand.

3) The current best fit data was used to start the process at ' 1 '.

This cycle was stopped when no improvement in fit was possible, and the mean absolute deviance between signal and output patterns was less than $5 \%$.

Table 1 The real world signal pattern, the accepted output pattern and the deviance of the output pattern from a perfect fit for the parameter value set chosen as the basis for all subsequent scenarios

\begin{tabular}{|c|c|c|c|}
\hline Output pattern & $\begin{array}{l}\text { Target value } \\
\text { (signal pattern) }\end{array}$ & $\begin{array}{l}\text { Accepted value } \\
\text { (output pattern) }\end{array}$ & Deviance \\
\hline $\begin{array}{l}\text { Proportion } \\
\quad \text { reproducers }\end{array}$ & 0.47 & 0.46 & -0.02 \\
\hline $\begin{array}{l}\text { Mean lifetime } \\
\text { offspring }\end{array}$ & 34.9 & 37.6 & 0.08 \\
\hline Young/litter & 4.8 & 4.8 & 0.00 \\
\hline Litters/female & 7.68 & 7.81 & 0.02 \\
\hline $\begin{array}{l}\text { Mean } \\
\text { reproductive } \\
\text { age }\end{array}$ & 712 & 706 & -0.01 \\
\hline $\begin{array}{l}\text { Maximum } \\
\text { reproductive } \\
\text { age }\end{array}$ & 2086 & 2061 & -0.01 \\
\hline $\begin{array}{l}\text { Male natal } \\
\text { dispersal }\end{array}$ & 0.93 & 0.89 & -0.04 \\
\hline $\begin{array}{c}\text { Female natal } \\
\text { dispersal }\end{array}$ & 0.64 & 0.62 & -0.03 \\
\hline $\begin{array}{l}\text { Mean breeding } \\
\text { density }\end{array}$ & 16.00 & 16.97 & 0.06 \\
\hline $\begin{array}{l}\text { Maximum } \\
\text { breeding } \\
\text { density }\end{array}$ & $>25$ & 35.61 & $\begin{array}{r}\text { test } \\
\text { passed }\end{array}$ \\
\hline $\begin{array}{l}\text { Minimum } \\
\text { breeding } \\
\text { density }\end{array}$ & 8 & 8.20 & 0.03 \\
\hline $\begin{array}{l}\text { Maximum } \\
\text { yearling } \\
\text { proportion }\end{array}$ & 0.6 & 0.62 & 0.03 \\
\hline $\begin{array}{l}\text { Minimum } \\
\text { yearling } \\
\text { proportion }\end{array}$ & 0 & 0.00 & 0.00 \\
\hline Correlation 1 & 0.77 & 0.75 & -0.03 \\
\hline Correlation 2 & -0.76 & -0.64 & -0.15 \\
\hline
\end{tabular}

Local Population Dynamics We selected signal patterns for POM development based on a set of literature data from studies conducted in Bayreuth, Germany [20-25]. These studies were carried in a 2-ha outdoor enclosure between 1988 and 1990. In total, 15 signal patterns were selected from these studies as detailed below and listed in Table 1:

- Natal dispersal: Dispersal was male-biased with $93 \%$ of juvenile males and $64 \%$ of juvenile females dispersing. Female dispersers most often moved to neighbouring territories whereas male dispersers moved further away. At the beginning of their first reproductive season, females that remained on their natal territories produced significantly more offspring than those that dispersed. Natal dispersal occurred during the first 5 months of life. The first breeding occurred in the following spring [20].

Natal dispersal was measured in males and females as whether after 5 months old they had moved from the warren that they were born in.

- Reproduction: Due to a postpartum oestrus [26], females can deliver up to 7 litters during the reproductive season, but the mean number of litters was only 3.2 per female per year [25]. Similar reproductive rates are recorded from rabbits shot from natural populations in England, Australia and New Zealand [27-29]. It is assumed that this lower than maximum fecundity is due to uterine losses of whole litters [26-32]. In the model, we link this phenomenon to weather and density-dependent constraints. Von Holst et al. [25] found that females gained a mean reproductive age of $712 \pm 37$ days (range 69-2086 days) and produced a mean of $7.68 \pm 0.52$ litters with $34.9 \pm 1.9$ offspring during their entire life. However, only $47.2 \%$ of all females had any reproductive success. In accordance with the results of other studies [33-36], the litter sizes varied between 1 and 9 with a mean of 4.8 offspring, although the mechanisms behind this are unknown. Proportion of reproducing females, lifetime litter production, mean and maximum reproductive age (measured as age from 300 days to death) and litter size were all selected as patterns.

- Density fluctuations: Rodel et al. [23] found that fluctuations of breeding density were paralleled by variations in the proportion 1-year-old females. They also found strong evidence for density-dependent response of individual reproductive effort. Two correlations were presented, the first between the number of 1-year-old females and breeding female density (Correlation 1), and the second between number of litters per female and breeding female density (Correlation 2). Correlation 1 was positive, $r$ approximately 0.77 , whilst Correlation 2 was negative, $r$ approximately -0.76 . These two correlation coefficients were selected as patterns. From the same study, mean, maximum and minimum breeding density, and maximum and minimum proportion of 1-year-old females were 
estimated from the graphical data presented and used as patterns.

Local Dynamics Simulation Scenario To match the original Bayreuth study as closely as possible, a uniform 2 ha was created using the dry semi-natural grassland polygon type in ALMaSS. The parameter RABBIT_WARRENFIXEDSIZE was altered to allow 16 warrens of one or two burrows in a grid form (the original study had 14 artificial warrens but some rabbits dug new burrows). Each run was started with 15 male and 15 female rabbits and run for 50 simulation years. The first 10 years were ignored during the analysis.

Landscape-Level Simulation To make the overall model useful, it needs to be extrapolated to larger, landscape, scales. Here, we considered landscape-scale rabbit population characteristics of warren locations, spatial variability in density, sex ratio and temporal patterns of reproduction.

The Bayreuth study rabbit density (up to 50 rabbits ha ${ }^{-1}$ ) appears to be in the middle range of what is found in the wild, with some studies reporting rabbit densities of 100 rabbits $\mathrm{ha}^{-1}$ [37-39]. An upper limit of $100 \mathrm{rabbits} \mathrm{ha}^{-1}$ was selected as the target for the best landscapes. Once the results from the local dynamics POM exercise were available, it was possible to calculate a minimum warren spacing based on the diameter of exclusive warren area needed to achieve $100 \mathrm{rabbits} \mathrm{ha}^{-1}$ in optimal uniform habitat. In heavy soils, this was found to be $55 \mathrm{~m}$, and for light soils $45 \mathrm{~m}$.

To predict rabbit densities, the distribution of warrens in a landscape is needed, but it is very difficult to find good signal patterns in general literature. The process of fitting parameters for warren distribution was therefore to use an expert judgement based on patterns expected from literature studies [e.g. 6, $40,41]$ and constrained by the maximum rabbit density. Locally, warrens should be spatially distributed showing patterns similar to available survey data (Appendix 1). This was done using 10 different landscape configurations, representing a range of agricultural landscapes from Denmark [42]. The best-fit parameter set for the local population dynamics was used as a starting point for fitting but two parameters were freely modified. These were the DISPERSALMORTPERM (extra probability of mortality per $\mathrm{m}$ during dispersal), and the maximum distance at which warrens are considered connected (MAXWARRENNETWORKDIST). These two parameters were chosen because in the fenced Bayreuth study, dispersal mortality was not expected to match that experienced by free rabbit populations and dispersal distance in the enclosure was small, preventing long distance dispersal and associated mortality.

POM fitting in this case was based primarily on weak patterns (spatial distribution of warrens, no exceedance of local density of 100 individuals /ha, spatial variation in warren occupancy). Initially, sex ratio seemed suitable as a strong signal pattern because it can be measured in the field. However, comparing different methods of population sampling indicates that there is considerable methodological bias [43], and therefore uncertainty regarding this pattern. In general, aboveground methods of 'capture' were biased towards males and within-warren methods towards females. If we assume withinwarren counts can be mapped to our within-warren population then the mean sex ratio was $0.688: 1(\mathrm{M}: \mathrm{F})$, with a lower extreme of $0.552: 1$ from [43] reporting on studies using traps or ferreting at the burrows. A similar result $(0.63: 1)$ was reported by Fernandez and Ceballos [44]. Aboveground captures suggested a male bias, with a maximum of $62 \%$ males [43]; therefore, the real value is likely to fall in between and is probably close to $1: 1$ or with a slight female bias.

The final signal pattern considered was the variation in timing of reproduction. This was a binary condition (true/false); the model should, as a minimum, re-create the range of between year changes in reproductive timing found in real world studies.

\subsubsection{Sensitivity Analysis}

Sensitivity analysis was primarily based on the Bayreuth study signal patterns where signal pattern uncertainty was low. This fitting involved 15 parameters and 15 output patterns (Figs. 1-15). A one-at-a-time sensitivity analysis was created by taking each parameter and adjusting it by \pm 25 , 50 and $75 \%$ of the chosen parameter fit from Table 1.

For sensitivity of landscape-scale parameters, the Himmerland landscape was selected as supporting a large but not extreme population of rabbits. To evaluate sensitivity of the sex ratio, spatial occupancy, CV of occupancy and mean population size, DISPERSALMORTPERM and MAXWARRENNETWORKDIST were varied by $\pm 25,50$ and $75 \%$ of the arbitrary starting point $(0.000133$ and 6.0$)$.

\subsection{Population-Level Impact Assessment for Rabbits and Hares}

\subsubsection{Landscapes}

Ten different landscape maps previously used for assessment of impacts of an endocrine disruptor on hares [42] were used for all the scenarios. Each landscape had a unique set of farms, farm types and arable, grassland and non-agricultural structures (see Appendixes 2 and 3 in Topping, Dalby et al. 2016). In all rabbit scenarios, we assumed the same weather conditions as used to simulate the Bayreuth experiment used for rabbit model development. All scenarios were run using assumptions of heavy and light soil types; hence, a total of 20 landscapes were used to evaluate each pesticide scenario. 


\subsubsection{Baseline Scenarios}

Baseline scenarios were run as 20 replicates of the same scenario length and conditions as the relevant toxicant scenario, differing only in the fact that a toxicant was not sprayed. No other change was needed since there was no indirect link between toxicant application and rabbits, i.e. we assume the plant protection product is an insecticide and therefore has no effect on rabbit food.

\subsubsection{Toxicant Effects}

Two types of toxicant effect were considered. Most scenarios were run with an endocrine disruptor (ED) effect, which caused litter loss by gestating females once they reached full term. Some scenarios were additionally run with a toxicant assumed to be acutely toxic, resulting in mortality of any rabbit exposed above a threshold body-burden.

\subsubsection{Toxicant Uptake}

Rabbits were assumed to feed from an area surrounding their warren of $200 \mathrm{~m}$ diameter. The maximum concentration method was used for ingestion; this assumes that the rabbits will feed from the forage with the highest concentration of toxicant available to them, with toxicant concentrations evaluated on a daily basis. This is a conservative assumption compared to, for example, the mean concentration method, which would assume that the rabbits eat equally from all possible forage areas and that the ingested toxicant corresponds to a mean concentration on forage that day.

Rabbits were assumed to eat $500 \mathrm{~g}$ of forage per day, and toxicant internal concentration was determined assuming $100 \%$ uptake of ingested toxicant. Five hundred grams forage intake per day was arrived at based on Cooke [45], who estimated daily food intake of a free-ranging wild lactating rabbit of $97 \mathrm{~g}$ dry matter per day. If we assume vegetation is approximately $80 \%$ water (based on EFSA [2]), wet weight would be close to $500 \mathrm{~g}$. Body burden was a function of ingested toxicant and body size. The proportion of internal toxicant eliminated was fixed and calculated on a daily basis.

Pesticide Application and Environmental Fate The application rate and the body-burden concentration that triggered an impact in rabbits were fixed in all scenarios. An environmental half-life $\left(\mathrm{DT}_{50}\right)$ of 7 days was assumed initially and spray drift was calculated up to $12 \mathrm{~m}$ from the edge of any sprayed field. In all cases, the maximum concentration method was used and no bioaccumulation was assumed. The application date for pesticide was 25th May in all cases unless otherwise stated.

\subsubsection{Scenarios}

Scenario 1: Comparison to Hare Impacts The aim of this scenario was to compare population impacts of the same toxicant (assumed to be an endocrine disruptor applied at the same rate and with the same toxicity per unit body weight) on both hare and rabbit populations using the same landscape structures and farming. The scenarios used by [9] were recreated for the rabbit using the $\times 10$ application rate of the original study. Model hare foraging differs from the rabbit in being simulated in 10-min time-steps and therefore toxicant intake varies with forage type in 10-min steps. The rabbit model was set to use the maximum concentration method, whereby the rabbit is constantly consuming the available forage source with the highest residues, and thus was more conservative than the hare. Thirty-year simulations were run with the toxicant applied in the last 10 years to all winter wheat and spring barley fields. In this scenario, pesticide application to spring barley was on April 30th and winter wheat applications on 15th May to match the original study.

Further Rabbit Scenarios A set of increasingly extreme situations were evaluated using the rabbit model. These were designed to demonstrate maximum potential effects that might be possible in the different landscapes by increasing exposure of all rabbits foraging from agricultural areas and by changing the assumed toxic effect of the pesticide.

These scenarios were all based upon the scenarios used to compare rabbit and hare impacts above, with toxicant applied to winter wheat and spring barley. All settings were as for these scenarios except the details noted below:

- Scenario 2: all fields in the landscapes were assumed to grow winter wheat which was sprayed with the endocrine disrupter at the same application rates used for Scenario 1. Note that here a new baseline was needed to compare rabbits in a landscape with all crops being winter wheat with and without pesticide.

- Scenario 3: identical to Scenario 2, except that the application rate was increased by a factor $10^{\wedge} 3$.

- Scenario 4: identical to Scenario 2, except that the application rate was increased by a factor $10^{\wedge} 6$.

- Scenario 5: identical to Scenario 2, except that the $\mathrm{DT}_{50}$ was increased to 76 days.

- Scenario 6: identical to Scenario 2, except that the $\mathrm{DT}_{50}$ was increased to 180 days.

- Scenario 7: identical to Scenario 2 except that we assume the effect of the pesticide is acute and immediate mortality.

- Scenario 8: identical to Scenario 7, except that the application rate was increased by factor $10^{\wedge} 3$.

- Scenario 9: identical to Scenario 7, except that the $\mathrm{DT}_{50}$ was increased to 180 days. 
- Scenario 10: was identical to Scenario 3, except that impacts on the hare were evaluated instead of the rabbit.

In all cases, 20 replicates were run for each scenario/ landscape combination. A power calculation was used, calculating the ratio of population size to $95 \%$ confidence interval of the population size estimate. In $80 \%$ of cases, power was 0.99 (95\% ci was $1 \%$ of the mean), and in the worst cases ( $1 \%$ of landscape/scenario combinations) the power was 0.95 .

\section{Results}

As noted in the methods, this study is in two parts. Sections 3.1-3.4 are the results of the POM process for developing the rabbit model, and section 3.5 the results of resulting model application to the landscape and pesticide scenarios.

\subsection{Model Development}

Using the local and landscape-level patterns, it was possible to develop a rabbit model able to replicate 13 out of 15 signal patterns to the desired accuracy (Table 1). Of the remaining two, mean lifetime offspring was $8 \%$ higher than recorded in the Bayreuth studies and the correlation between number of litters per female and breeding female density (Correlation 2) was too low $(r-0.64 \mathrm{cf} r$ of -0.76$)$. All other population descriptors were within the $5 \%$ tolerance.

The full description of the resulting ALMaSS rabbit model is in ODdox format [10] and is available online. ${ }^{1}$ The ODdox contains an overview section and links directly to the $\mathrm{C}++$ code used to run the model. The code is open source and available from GitLab. ${ }^{2}$ Nevertheless, a very short description of the model is presented here to aid reading of the current paper.

\subsection{Rabbit Model Overview}

General Individuals in the rabbit model are modelled as five different types of objects, which are defined as classes. Four classes represent kits, juvenile and male and female rabbits, and the fifth is used to manage the warren as a collective. This means that the individual rabbits are classified as being either 'Young', 'Juvenile', adult 'Male' or adult 'Female'. Each class has a number of attributes and behaviours associated with it and these attributes may be transferred between classes, e.g. as young mature to become juveniles, or juveniles to adult then their age and lifetime nutrition is transferred.

The model is initiated by randomly 'seeding' the landscape with adult male and female rabbits that must find a warren

\footnotetext{
${ }_{1}^{1}$ https://almassdocs.au.dk/ALMaSSODdox/Rabbit/index.html

${ }^{2}$ https://gitlab.com/ChrisTopping/ALMaSS_all
}

with free burrow capacity before establishing themselves as breeding pairs of rabbits. At each time-step, adult rabbits will assess their status in terms of mates, burrows and dominance. Young model rabbits are dependent on their mother until weaned, at which point they are considered juvenile and act as independent rabbits.

Dispersal If it is possible to move to a nearby warren to improve status then the rabbit can do this, but only if that warren has been explored previously. Dominance alters the chance of successful litter production as well as the chance of mortality (dominant animals are assumed to live longer and have a higher chance of breeding successfully).

Habitat Quality The number of burrows in a warren is determined by soil type and habitat quality, and is therefore the primary density-dependent controlling factor, since once all are occupied the breeding population is at maximum. Habitat quality is determined by the total amount of suitable forage habitat available within the forageable area around the warren.

Dispersal Juvenile and adult rabbits can explore warrens close to them, if they are within a fixed distance and juveniles in particular will attempt to disperse to nearby warrens if it is possible to find a mate or burrow. Adults are much less likely to move and will be more likely to occupy any vacant burrows within the current warren when they become available.

Social Status Dominance is determined by age and burrow status. It influences the likelihood of successful breeding, mortality and dispersal.

Reproduction A female rabbit that comes into oestrous and has a mate will automatically become pregnant. Onset of first oestrous per season is assumed to be controlled by temperature over the previous month. Similarly, cessation of breeding is determined by temperature in the autumn. If the female is lactating when becoming pregnant, then the young are forced out of the burrow at 30 days, at which point she can give birth again; the likelihood of successful births increases with social status and age.

Foraging and Growth Successful foraging is assumed to be a function of the weather (temperature and rainfall). This is controlled by parameters MINBREEDINGTEMP and MINFORAGETEMP that together alter the number of forage days possible in any given weather year. Growth is assumed to occur only on suitable forage days, which are summed as a measure of lifetime nutritional status. On each suitable day, the rabbit forages from the warren area and as a result may be exposed to pesticide. Exactly how much pesticide is ingested depends on the assumptions regarding foraging behaviour. 
Two basic defaults can be selected: (1) that the rabbit will forage from all suitable forage locations equally, and therefore will obtain an average dose based on the concentration of residue in the forage areas, or (2) that it will forage from the area where it receives the maximum dose. Suitable forage locations from which the rabbits are allowed to forage on any given day depend on vegetation type, height and digestibility.

Mortality All rabbits are subject to a daily mortality chance; the magnitude of this depending on age, lifetime nutritional status, social status and disease prevalence. To simulate disease, we need a globally varying probability with local variations depending upon density. This is done by evaluating two levels; the first is the total population density. This is given by the total number of rabbits divided by the total number of warrens. The second is the local density, which is the number of rabbits related to the warren carrying capacity. These two factors are combined to create an overall disease mortality chance. Calculation of the disease mortality chance is carried out at intervals throughout the year (DENDEPPERIOD in Table 2). Rabbits will also die if they are subjected to external events (e.g. acute poisoning) or if they reach the end of their natural life span which is set as being 4.5 to 6.5 years.

\subsection{Sensitivity Analysis}

\subsubsection{Local Population Dynamics}

Due to the large number of parameters a one-at-a-time sensitivity analysis was performed on the parameters important for fitting to the Bayreuth study real world patterns. Each parameter was varied by $+/-25,50$ and $75 \%$ of its value in Table 2 . The proportion change in 13 output patterns for all 17 parameters used for fitting is shown graphically in Appendix 2, together with short notes on what the parameter does in the model. They are also summarised in Fig. 1, below. There were some parameters which elicited strong responses when varied and others to which the model is largely or wholly insensitive. Therefore, the sensitivity values for each parameter in Table 2 are a guide only, and particular patterns may be highly sensitive to parameters that generally affect the model output patterns less and vice versa; hence, some judgement is needed.

All values are fitting parameters. This means that there are no literature values available to transfer to these parameters directly. However, those parameters with real world counterparts, e.g. the minimum temperature for foraging, fall within sensible values. The main determinant of the uncertainty of all these parameters is therefore the reliability of the real world signal pattern to which they are fitted and the degree to which that pattern is matched.
Table 2 The parameters varied in this part of the POM fitting and the final values selected to create the fit in Table 1

\begin{tabular}{|c|c|c|}
\hline Parameter name & Value & Sensitivity \\
\hline ADULTBASEMORT & 0.00013 & Medium \\
\hline DENDEPPERIOD & 90 & Medium \\
\hline DENSITYDEPSCALER & 0.45 & Medium \\
\hline DIGGINGTIME & 10 & Low \\
\hline DISEASEDENDEPDAY & 53 & High \\
\hline DISPERSALMORTPERM & 0.000005 & Low \\
\hline FORAGEAREAMORTSCALER & 6 & Medium \\
\hline GLOBALDISEASEPROBCONSTANT & 0.05 & Medium \\
\hline JUVENILEBASEMORT & 0.004 & Medium \\
\hline LITTERABSOPRTIONCONST & 1.8 & High \\
\hline MAXFORAGEHEIGHT & 30 & Low \\
\hline MAXFORAGERAINFALL & 3.6 & Low \\
\hline MAXKITS & High & High \\
\hline MINBREEDINGTEMP & 330 & Medium \\
\hline MINFORAGETEMP & -0.83 & Low \\
\hline SOCIALREPROTHRESHOLD & 2.3 & Medium \\
\hline YOUNGBASEMORT & 0.038 & Low \\
\hline
\end{tabular}

These values form the centre-point from which the sensitivity analysis was based. Sensitivity indication are summarised from Fig. 2 as low sensitivity $<0.01$ overall fit deviation over the range $0.5-1.5( \pm 50 \%)$, high sensitivity $>0.05$ over the same range. The parameter names are those used in the code and the function is fully described in the ODdox documentation https://almassdocs.au.dk/ALMaSSODdox/Rabbit/index. html

\subsubsection{Landscape-Level Patterns}

Landscape-level patterns were relatively insensitive to changes in the two parameters evaluated at this scale (Fig. 2), and predicted sex ratio was also insensitive. There is an indication of an interaction at high dispersal mortality: the response range to changes in maximum network distance approximately doubled compared to low dispersal mortality.

\subsection{Landscape-Level Patterns of Density and Occupancy}

The pattern of occupancy differed greatly between landscapes. Figure 3 shows some examples of overall and local distribution of warrens. Summary statistics are graphed for all 10 landscapes, and show large variation in terms of landscape occupancy, mean population size and mean density. These fall within ranges of rabbit density reported in the literature. In all cases, assuming light soil generated lower population sizes and lower proportions of warrens occupied than assuming heavy soil, whereas the absolute number of occupied warrens and landscape-level occupancy was always higher in light soil (due to the higher total number of warrens, occupied and unoccupied, but with lower average number of individuals in each occupied warren). 
Fig. 1 The overall model fit plotted against change in parameter value for 17 parameters tested in the local population dynamics sensitivity analysis. The $y$-axis is constrained to 0.0 0.1 for clarity. See Appendix 2 for an explanation of each parameter

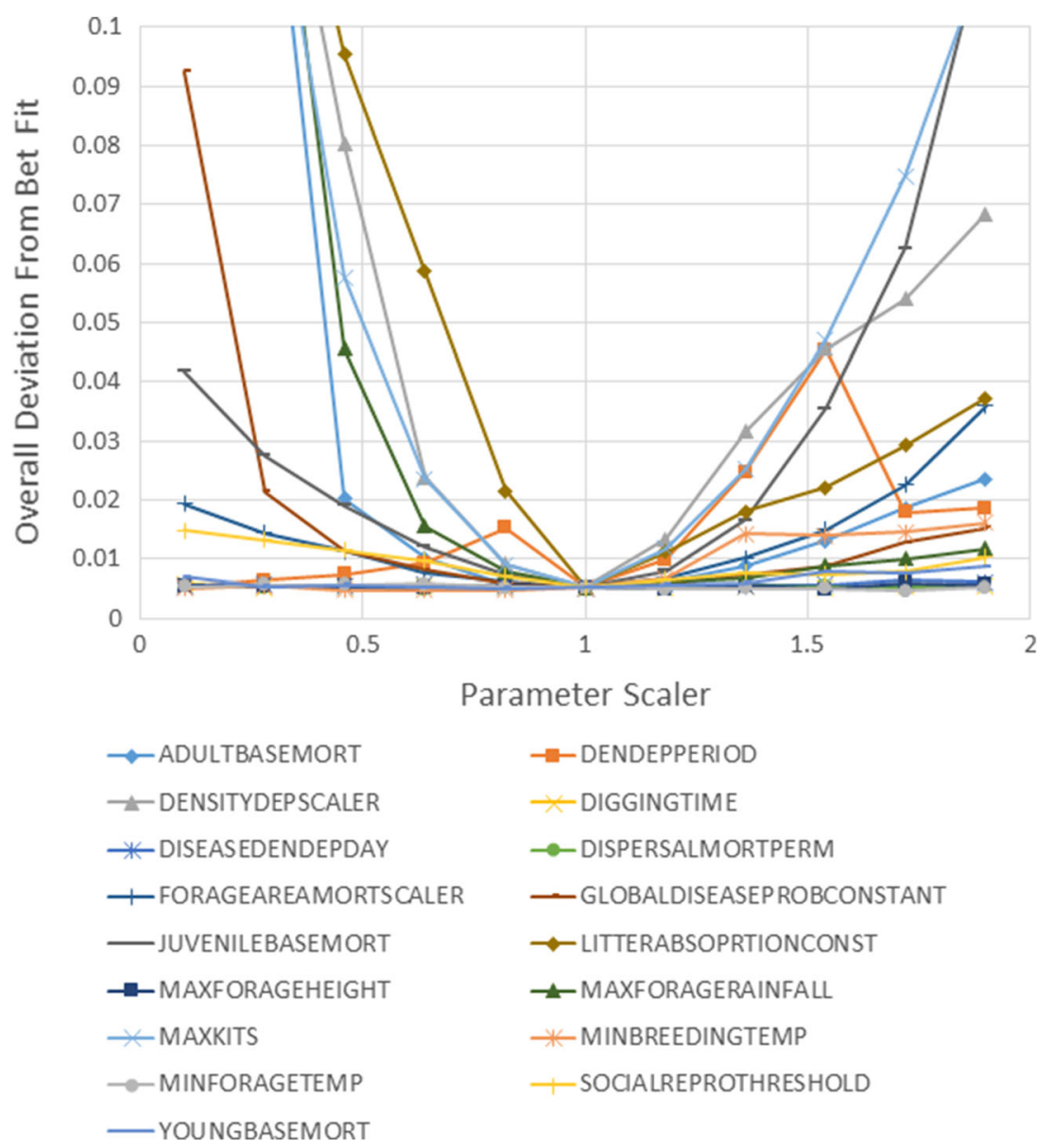

\subsubsection{Phenology of Litter Production.}

The actual patterns of kit numbers for 1994-1999, which have most of the extremes, are graphed below (Fig. 4). For comparison to field data on kit numbers, the extreme dates of these annual distributions should probably be ignored, because in field surveys it is not likely that the very first and very last litter could be observed; hence, for comparison to field values for kit numbers, we used values from the initial high to the point of decline at the end of season. Table 3 shows the first date of litter production and the last date for Tønder landscape using Bayreuth weather data for 1993-2002, maximum range 1st March to 19th October. Tønder was chosen because it supports the highest density of rabbits and has large areas of suitable grassland habitat, which are less disturbed by agricultural production, therefore was most similar to the enclosure study. The enclosure study [25] showed ranges of reproductive season from March 9th to October 20th, fitting quite well with the early peak in litter production, but with a shorter season than in the model. The variation in breeding season length also concurs with [46], who found reproductive periods varying amongst seasons with different weather. In their study in East Anglia UK, the first female oestrus was advanced by up to 1 month and young emerged over an extra month in good years, with their first appearance above ground advanced by 2 months.

\subsection{Effects of Toxicants}

Scenario-1 baseline rabbit populations were very different in the different landscapes but impacts of the toxicant on the rabbit were minimal. We used the abundance occupancy index, or AOR-index, [47] to compare to the original hare impacts. This index shows the relative change in occupancy in terms of proportion of the landscape occupied, and the change in density of the areas occupied, all relative to a baseline. A rough estimate of total effect can be made by summing the two AOR index scores. This index showed that population effects on hares (compared to rabbits) were typically greater than 10 times on abundance, with a maximum impact of $-50 \%$. Except for the Mors landscape (-1\%), there was no impact on rabbit occupancy in either soil type, but impacts on hares up to $-32 \%$ (Table 4).

Exposure of all rabbits in the landscape to the Scenario 1 toxicant had no noticeable impact on the summed AOR scores of the rabbit populations (Table 5). Increasing application rate by a factor $10^{\wedge} 3$ and $10^{\wedge} 6$ did not increase the impact noticeably for the endocrine disruptor (Sc.3 and 4) and nor did 


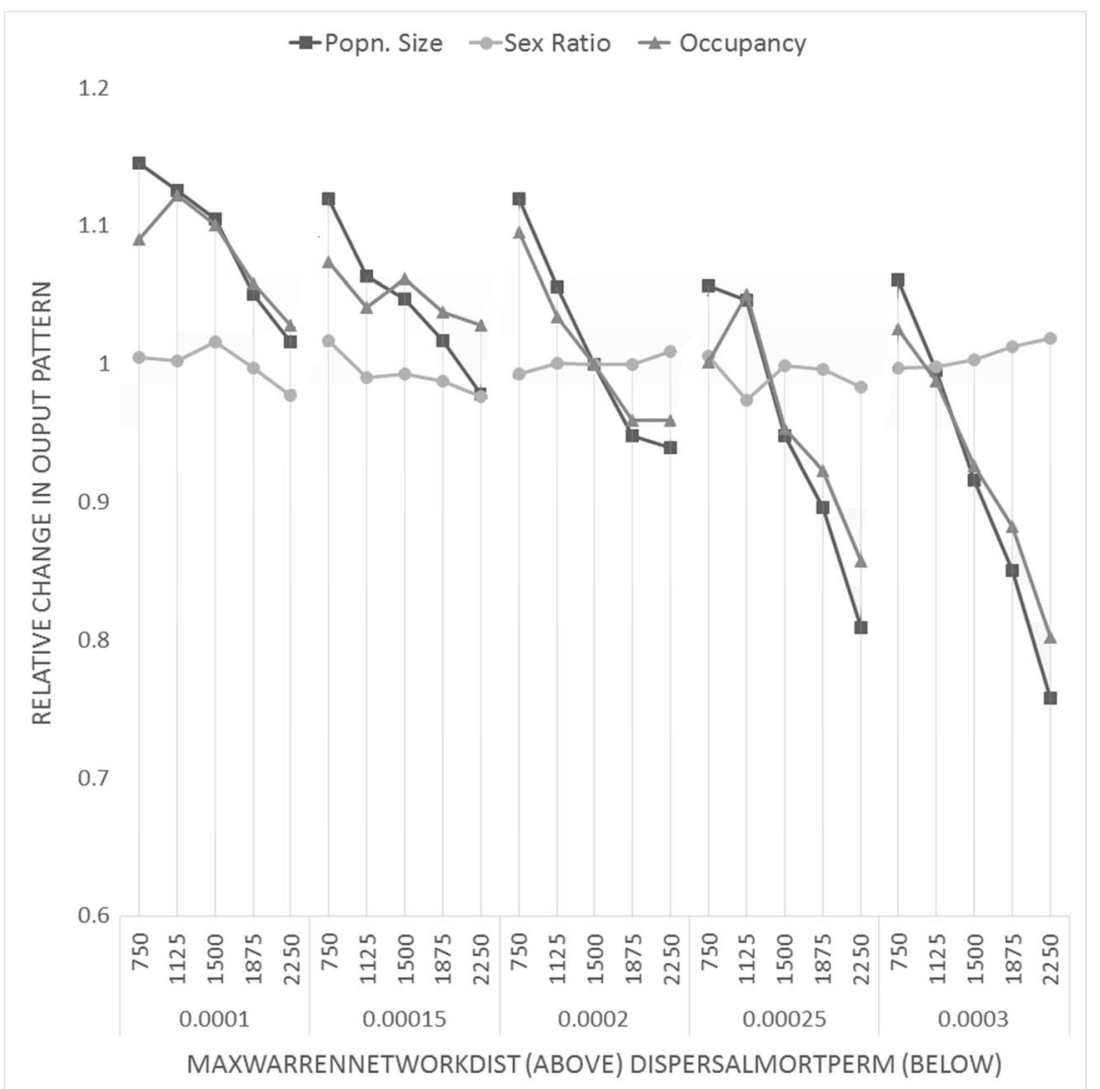

Fig. 2 Output pattern response of mean population size, sex ratio and occupancy for rabbit populations in the Himmerland landscape when varying the parameters MAXWARRENNETWORKDIST and DISPERSALMORTPERM

extending the $\mathrm{DT}_{50}$ to 76 days (Sc.5), but a $\mathrm{DT}_{50}$ of 180 days decreased AOR scores by -0.03 to -0.20 (Sc.6). In contrast, acute mortality (Sc.7-9) had large impacts even at the standard rate of application, increasing with application rate and reaching summed AOR reductions of 0.12 to 1.44 in Scenario 9 (Table 5).

Landscapes had a large impact. This is most clear from the scenarios with high impact, and trends are consistent between scenarios. Impacts on Næstved, Lolland and Esbjerg were always high, and very low impacts in Tønder (Tn). A comparison of summed AOR scores between heavy and light soil scenarios showed little difference in low impact scenarios, but in Scenario 6 (ED, $\left.\mathrm{DT}_{50} 180 \mathrm{~d}\right)$ and the acute toxicant scenarios (7-9), there were differences. In Scenario 6 (ED), impacts were all greater in light soil scenarios, whilst the pattern was varied for the acute toxicant scenarios with no clear trend (Table 6).

When comparing the impact of ED and acute toxicant scenarios, there were clear differences in the spatial impact of the two modes of action. In scenario 6 (ED), the mean abundance across all landscapes was $-14 \%$, the mean occupancy $-1 \%$ and population size change was $-15 \%$. The same statistics for scenario 9 (acute) were $0,-75$ and $-74 \%$ (not shown). Density, where the rabbit population persisted, was therefore not affected by the acute toxicant scenarios; but although the ED scenarios reduced density, the distribution was much less affected.

Scenario 10 results (hare; $\times 1000$ application rate of ED; equivalent to rabbit Scenario 3) were unequivocal: hare populations were driven to extinction or near extinction in all landscapes.

\section{Discussion}

An ALMaSS-based model of landscape-scale rabbit population dynamics was successfully made, though with some remaining important uncertainties. These are as follows:

- There is a need to verify the spatial distribution of potential warrens on a much larger scale than is currently possible, since landscape patterns used to fit the model are characterised as 'weak' patterns, meaning that they are generalities rather than precise targets.

- Dispersal distances and mortalities are estimated from literature and the landscape patterns. These values are highly uncertain, although the model is relatively insensitive to these parameters. 
(a)

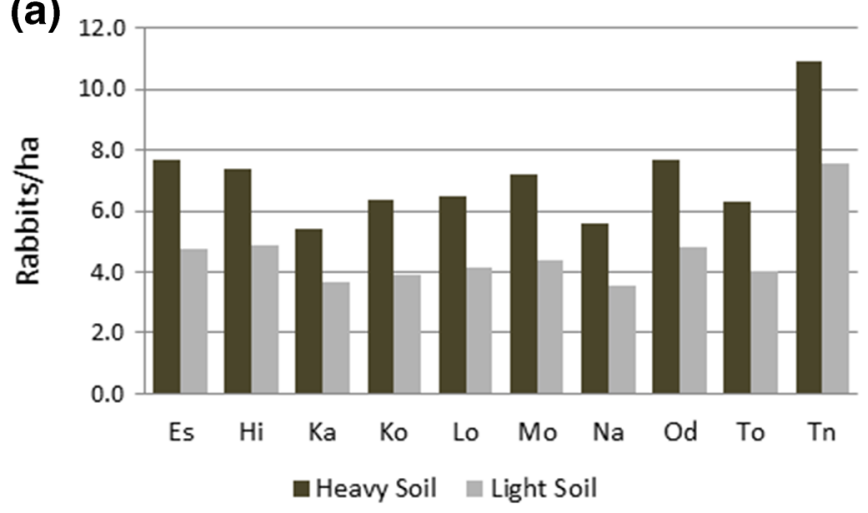

(c)

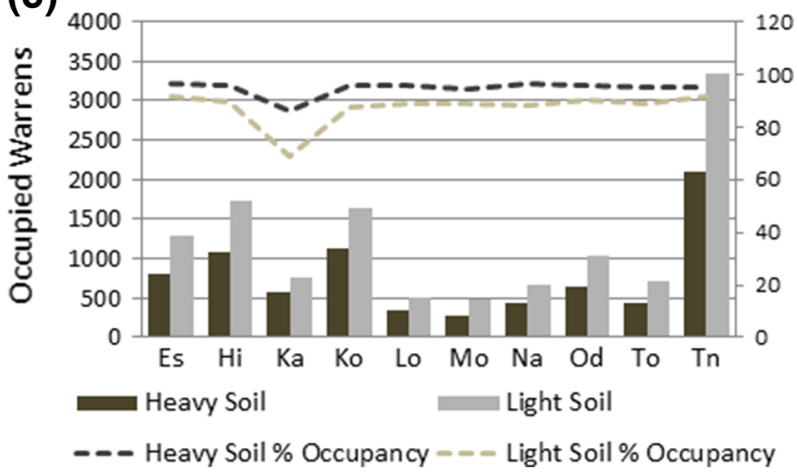

Fig. 3 Four statistics describing rabbit populations under standard agricultural conditions with no toxicant added and for assumptions of light and heavy soil. The landscape was divided into a regular grid of $100 \mathrm{~m} \times 100 \mathrm{~m}$ and rabbits recorded within these grid cells and per warren, and in total. a Mean density of rabbits (rabbits/ha) for all 1 ha grid cells where rabbits were present. $\mathbf{b}$ The number of 1 ha grid cells

- Of the parameters to which the model was most sensitive, the most influential are density-related effects and were fitted based on the Bayreuth study. The uncertainty regarding these is therefore linked to the extent to which that study can be taken to represent rabbit ecology in (b)

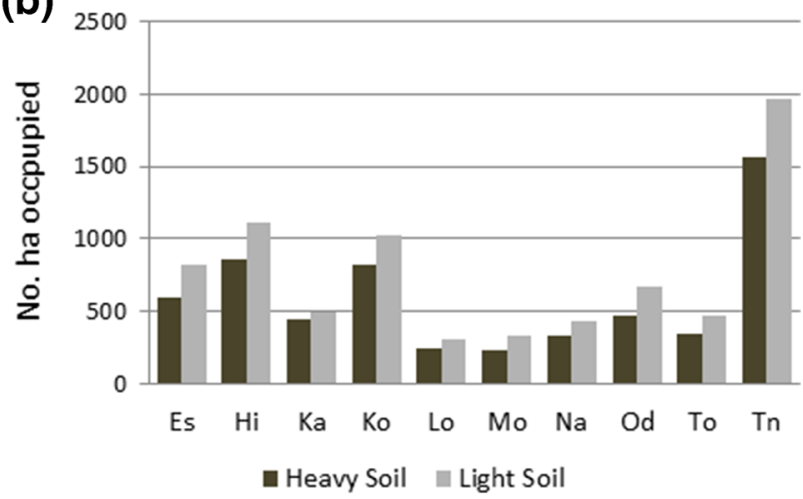

(d)

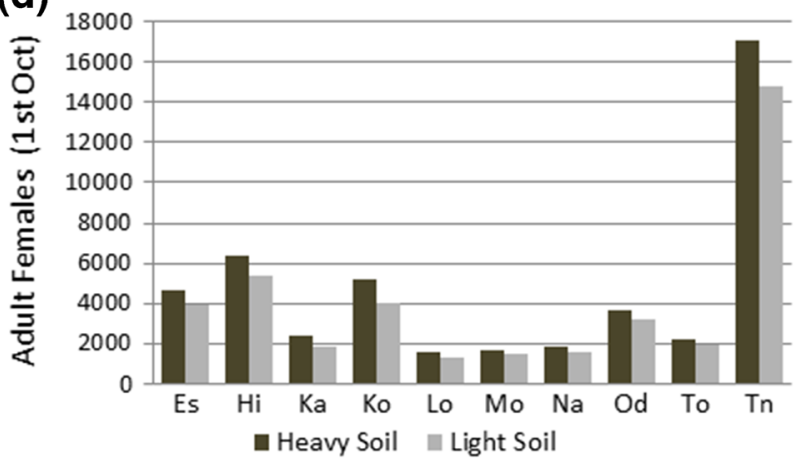

occupied by rabbits. c The mean number and proportion of potential warrens that were occupied annually. d Mean adult female annual population size on 1 st October over the final 10 years of a 30 -year simulation. X-axis codes refer to different landscapes: Es Esbjerg, Hi Himmerland, Ka Karup, Ko Kolding,Lo Lolland, Mo Mors, Na Næstved, Od Odder, To Toftlund and Tn Tønder landscapes

general. Other sensitive parameters have a more predictable range. For example, the maximum number of kits per litter and the maximum forage rainfall were important, but are not likely to vary to the extent tested in the sensitivity analysis.
Fig. 4 Model kit abundance based on 1994-1999 weather data from Bayreuth Germany (which included most extremes in phenology) using the Tønder landscape

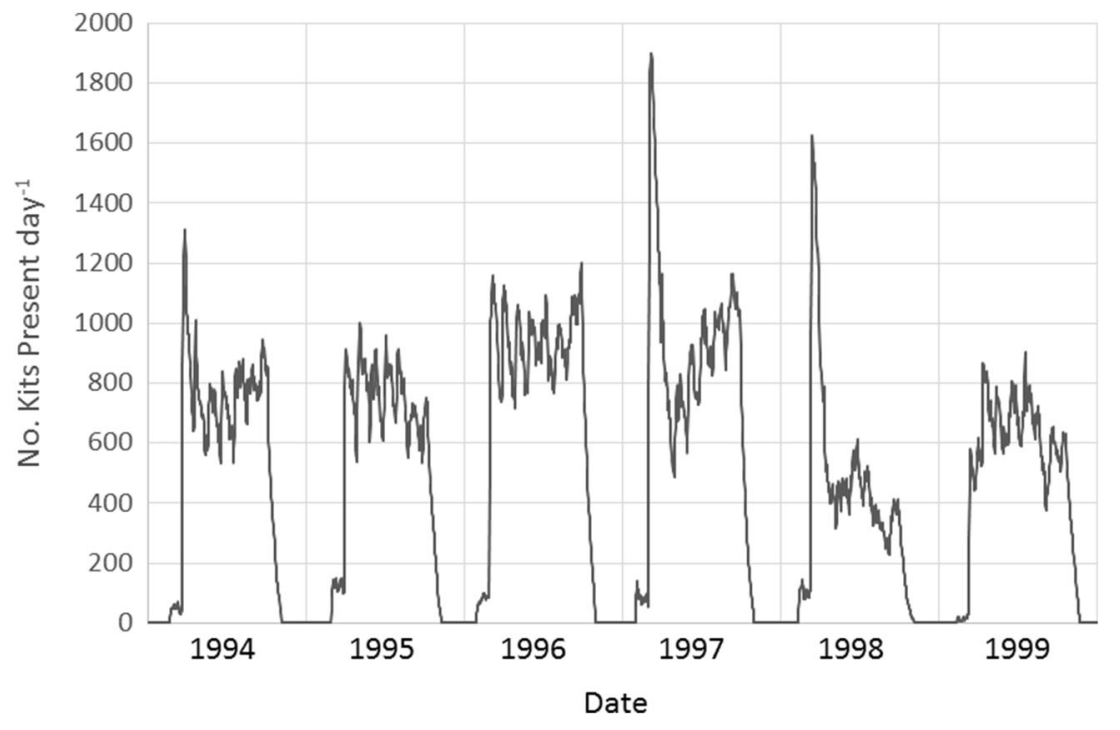


Table 3 First and last date of peaks in occurrence of kits during baseline simulation runs in Tønder landscape for weather years 1992 2001

\begin{tabular}{lll}
\hline Year & Start & Stop \\
\hline 1992 & 25-Mar & 22-Sep \\
1993 & 20-Mar & $26-$ Oct \\
1994 & $27-$ Mar & $01-$ Oct \\
1995 & 03-Apr & $03-$ Oct \\
1996 & 05-Mar & $27-$ Sep \\
1997 & 01-Mar & $27-$ Sep \\
1998 & 11-Mar & $25-$ Sep \\
1999 & 10-Mar & $19-$ Oct \\
2000 & 17-Mar & $15-$ Oct \\
2001 & 24-Mar & $26-$ Sep \\
\hline
\end{tabular}

- Background predation levels are assumed to be density independent and constant in time. This is unlikely to be accurate; however, there is little evidence of predation being an important population control factor [48].

- We used Danish landscapes and farming but, with the exception of southern Denmark and islands, rabbits do not occur in these landscapes. We therefore used the weather data from central Germany and assumed that the level of predation used was typical of central Europe. This leaves the potential for differences in farm activities and detailed landscape structure to affect the results. However, given the range of landscapes tested, any effect of this is not likely to be high.

Nevertheless, model fit to the patterns from the Bayreuth field enclosures study was reasonable, and model results and behaviour were commensurate with available literature data. Therefore, we expect the model to predict effects of toxicants on a rabbit population reasonably well within a domain of applicability of central to northern Europe.
Putting aside the remaining uncertainties, the rabbit model indicated that it is generally difficult to drive a rabbit population to extinction, though strong effects can occur in certain circumstances. An acute toxicant had a much greater effect on rabbit populations than an endocrine disruptor affecting reproduction. It should be noted that both toxicants were extreme and unrealistic imaginary cases.

Environmental fate of the toxicant has a large effect, as shown by a comparison of scenarios 2 to 6 (ED scenarios). Increasing the application rate in Scenario 2 (DT 507 days) had little or no effect because, even in the case of $10^{\wedge} 6$ times the initial rate, the decay function results in toxicant levels below the critical threshold before rabbit breeding starts. Increasing the $\mathrm{DT}_{50}$ to 180 days (Scenario 6), so that environmental toxicant concentrations still cause an effect the following May, increased impacts from $4 \%$ to up to $20 \%$. This did not, however, stop model rabbits from refuge habitats dispersing to agricultural warrens and attempting to breed. In fact, these rabbits had high survival rates because of low densities of the young. In contrast, we see a more dramatic effect of acute mortality, which removes all foraging rabbits immediately. However, it is interesting to note that density in non-exposed areas remains high, suggesting in this case there is no significant depletion effect, or 'action at a distance' [49] seen in other systems [50, 51].

The initial comparison between the rabbit and hare population vulnerability indicated that the hare population was much more vulnerable to the same toxicant even though the toxic impact would be approximately four times lower due to the higher body mass. In addition, the hare model used a detailed daily foraging algorithm resulting in a precise estimate of pesticide intake, whereas the rabbit model more conservatively assumed $100 \%$ of ingested matter to be contaminated at the concentration of the most toxicant-contaminated area within the warren foraging area. Despite this conservative assumption for the rabbit, it was not possible to drive the population to extinction using an endocrine disruptor

Table 4 AOR index scores and overall population size changes measured for hare and rabbit population under Scenario 1 conditions for heavy and light soil assumptions in 10 landscapes (Es-Tn)

\begin{tabular}{|c|c|c|c|c|c|c|c|c|c|c|c|}
\hline Landscape & & Es & $\mathrm{Hi}$ & $\mathrm{Ka}$ & Ko & Lo & Mo & $\mathrm{Na}$ & Od & To & $\operatorname{Tn}$ \\
\hline \multirow[t]{2}{*}{ Hare } & Ab. & -0.22 & -0.23 & -0.18 & -0.34 & -0.16 & -0.48 & -0.50 & -0.42 & -0.37 & -0.05 \\
\hline & Occ. & -0.15 & -0.14 & -0.03 & -0.19 & -0.32 & -0.05 & -0.08 & -0.13 & -0.04 & -0.13 \\
\hline \multirow[t]{2}{*}{ Rabbit (heavy soil) } & $\mathrm{Ab}$. & -0.01 & -0.01 & -0.01 & -0.01 & -0.02 & -0.01 & 0.00 & 0.00 & -0.01 & 0.00 \\
\hline & Occ. & 0.00 & 0.00 & 0.00 & 0.00 & 0.00 & -0.01 & 0.00 & 0.00 & 0.00 & 0.00 \\
\hline \multirow[t]{2}{*}{ Rabbit (light soil) } & $\mathrm{Ab}$. & -0.01 & 0.00 & 0.00 & 0.00 & -0.01 & 0.00 & 0.00 & 0.00 & 0.00 & 0.00 \\
\hline & Occ. & 0.00 & 0.00 & 0.00 & -0.01 & 0.00 & 0.01 & 0.00 & 0.00 & 0.00 & 0.00 \\
\hline \multicolumn{12}{|c|}{ Overall population reduction } \\
\hline \multicolumn{2}{|c|}{ Hare } & 0.33 & 0.34 & 0.21 & 0.46 & 0.43 & 0.51 & 0.54 & 0.50 & 0.39 & 0.17 \\
\hline \multicolumn{2}{|l|}{ Rabbit (heavy soil) } & 0.01 & 0.01 & 0.01 & 0.01 & 0.02 & 0.01 & 0.01 & 0.01 & 0.00 & 0.00 \\
\hline \multicolumn{2}{|l|}{ Rabbit (light soil) } & 0.01 & 0.00 & -0.01 & 0.01 & 0.01 & -0.01 & 0.00 & 0.00 & 0.00 & 0.00 \\
\hline
\end{tabular}


Table 5 Summed rabbit AOR scores for all scenario/landscape combinations and heavy soil

\begin{tabular}{|c|c|c|c|c|c|c|c|c|c|}
\hline Landscape & Sc.1 & Sc. 2 & Sc. 3 & Sc. 4 & Sc. 5 & Sc. 6 & Sc. 7 & Sc. 8 & Sc. 9 \\
\hline Es & -0.01 & -0.02 & -0.02 & -0.03 & -0.03 & -0.20 & -0.28 & -0.63 & -1.13 \\
\hline $\mathrm{Hi}$ & -0.01 & -0.02 & -0.02 & -0.03 & -0.03 & -0.17 & -0.20 & -0.37 & -0.62 \\
\hline $\mathrm{Ka}$ & -0.01 & -0.01 & -0.02 & -0.03 & -0.03 & -0.18 & -0.24 & -0.51 & -0.64 \\
\hline Ko & -0.01 & -0.01 & 0.00 & -0.02 & -0.02 & -0.13 & -0.17 & -0.46 & -0.97 \\
\hline Lo & -0.02 & -0.02 & -0.02 & -0.02 & -0.02 & -0.20 & -0.35 & -0.70 & -0.71 \\
\hline Mo & -0.01 & -0.01 & -0.01 & -0.02 & -0.02 & -0.12 & -0.16 & -0.30 & -0.44 \\
\hline $\mathrm{Na}$ & -0.01 & -0.01 & -0.01 & -0.02 & -0.02 & -0.17 & -0.27 & -0.62 & -1.44 \\
\hline Od & -0.01 & -0.01 & -0.01 & -0.02 & -0.02 & -0.15 & -0.17 & -0.39 & -0.59 \\
\hline To & 0.00 & 0.00 & -0.01 & -0.01 & -0.01 & -0.17 & -0.21 & -0.56 & -0.85 \\
\hline Tn & 0.00 & 0.00 & 0.00 & 0.00 & 0.00 & -0.03 & -0.02 & -0.06 & -0.12 \\
\hline
\end{tabular}

(reproductive effect) applied to agricultural areas, even when it was present at toxic levels throughout the year. In contrast, the hare population was fragile in all landscapes with the exception of Tønder, and was driven to extinction in an ED scenario where rabbit population-level reductions as combined AOR scores were 0.0 to -0.03 in all landscapes (Table 5, Sc.3). Impacts of acute immediate mortality (Scenario 7) on the rabbit were higher than the ED scenarios, and it was possible to reduce rabbit populations by over $90 \%$ (not shown), but still not to extinction. In the worst case, landscape occupancy of the rabbit was reduced by up to $94 \%$ compared to $<4 \%$ for the endocrine disruptor (not shown).

To explain these results, it is necessary to consider the interactions between the rabbit and hare spatial dynamics and the landscape structure used in the scenarios. In all cases, impacts were lower in the Tønder landscape than in all others. This is due to the large area of permanent grassland in this landscape, which provides habitat and refuge from exposure to the toxicant (assumed to be applied to arable fields only).

Table 6 Summed AOR scores for light soil subtracted from the same scenario summed AOR score for heavy soils for all ten landscapes

\begin{tabular}{lllll}
\hline & Scenario 6 & Scenario 7 & Scenario 8 & Scenario 9 \\
\hline Es & -0.06 & 0.00 & -0.03 & -0.09 \\
$\mathrm{Hi}$ & -0.04 & 0.01 & 0.02 & 0.01 \\
$\mathrm{Ka}$ & -0.04 & 0.04 & -0.01 & -0.07 \\
$\mathrm{Ko}$ & -0.02 & 0.01 & 0.05 & 0.00 \\
Lo & -0.04 & -0.03 & -0.03 & 0.22 \\
$\mathrm{Mo}$ & -0.03 & 0.01 & 0.03 & 0.06 \\
$\mathrm{Na}$ & -0.02 & 0.01 & -0.05 & 0.02 \\
$\mathrm{Od}$ & -0.04 & 0.01 & 0.01 & 0.05 \\
$\mathrm{To}$ & -0.05 & 0.02 & -0.02 & -0.01 \\
$\mathrm{Tn}$ & -0.01 & 0.01 & 0.01 & 0.04 \\
\hline
\end{tabular}

This is true for both rabbit and hare. The higher impacts on the hare, even in this landscape, were due to the spatial dynamics of hares versus rabbits. Rabbits exist in semi-permanent warrens, which effectively act as breeding territories. Rabbits also have high reproductive rates and typically a self-limiting population size (see model development). Warrens in nonexposed habitat therefore act not only as refuges but as sources of colonisers that can repopulate exposed warren sites. The hare does not behave in this way and will move around to obtain good forage conditions, having no fixed territory. Thus, even in Tønder, many hares will venture into the arable fields at some time and be affected by the toxicant.

These results should probably not be surprising. There is a large body of literature on rabbit control, which is notoriously difficult. Smith et al. [52] estimated that $£ 5$ million were spent annually on rabbit control in the UK; and in other countries, especially Australia and New Zealand, rabbits are considered both as pests and as a danger to endemic wildlife. Given the vigorous control campaigns in force it would be a surprise if rabbit populations were vulnerable to the use of field sprayed toxicants. In contrast, the brown hare has been in widespread decline throughout Europe since the 1960s [53] and is listed under Appendix 3 of the Bern Convention in Europe [54]. Declines are generally thought to be linked to habitat loss, primarily through agricultural intensification [55]. It is clear that the hare is a population under 'stress', and thus likely to be vulnerable to further stressors.

The results illustrate how the model can be used to investigate reasons behind differences in species' vulnerability, and to check for conditions under which vulnerability might occur in a species, for both protection or control programs. To do this, it is essential that the baseline conditions are carefully simulated and are representative of the domain of applicability that the model will subsequently be used in.

We note that other parameter fitting methods could have been used as part of the POM process (e.g. Bayesian 
approaches to fitting [56] or using the Elementary Effects method [57] for screening prior to fitting); it is perhaps unlikely that these would find a significantly better fit, but they may have been more time efficient. There are also other methods of developing models that share similarities with POM, such as pattern-guided evolution [58] and context-oriented model validation [59]. The latter being very similar, based on POM but with a more formal structure. All these methods are relatively new, and it will require more work and tool development before standards can be found, if this is possible at all.

The primary foci of this study were on testing the resilience of rabbit populations and comparing the rabbit and hare in regulatory risk assessment. Currently, regulatory risk assessment is done on a case by case basis considering a single PPP in isolation from all others [1, 2]. For the assessments carried out here, toxicity was set extremely high and therefore other stressors are unlikely to be of concern. However, in the real world, with more realistic toxicity, this situation is unlikely to apply and non-target organisms can be exposed to more than one PPP due to multiple applications over time and/or mixture application. In addition, the assumption of immediate degradation/excretion of PPPs is not justified, as bioaccumulation can occur in some cases. Since the current regulatory risk assessment lacks some aspects of realism, these factors may be taken into account in future regulatory risk assessments. This is already being considered, referred to as a systems approach [60], in which case, predictive integrative models such as presented here will be particularly valuable.

\section{Conclusions}

For EU risk assessment of plant protection products, these results clearly indicate that if the protection goal is population-level impacts, either in abundance and/or distribution, then the hare is a much more vulnerable species than the rabbit under all the conditions tested. Rabbits would only be more vulnerable than hares if the entire population were to be exposed simultaneously, when lower body mass would then be a critical factor. This did not occur even though the toxicant and exposure scenarios tested here were unrealistically extreme.

As well as specifically answering the question on rabbit versus hare vulnerability, this study generally illustrates the potential usefulness of models in setting focal species for risk assessments. Since developing and using these models is a specialised activity, the next steps towards developing population-level risk assessment could be for modelling specialists to develop standardised and accepted models, without the need for detailed review of each subsequent application, with user-friendly interfaces thus enabling standard use by non-specialists. This would need to be an iterative process in collaboration with risk assessors and risk managers. The result could be development of detailed focal species models to be used to determine protection goal metrics by back-calculation of acceptable population impacts to acceptable toxicity/use profiles; whereby these profiles could also be used in simple first tier risk assessments. This would help remove one of the biggest obstacles to model acceptance in ERA, i.e. the need for specialist risk manager knowledge of individual models. Of course the models could be further refined as more data become available, for example in the case of the remaining uncertainties discussed above for the ALMaSS rabbit model, or in the case of specific toxicant or landscape scenarios.

Acknowledgements Thanks to Rifcon $\mathrm{GmbH}$ for supplying the survey diagrams shown in Appendix 1. Thanks also to Graham Smith and David Cowan for their helpful discussions on rabbit ecology during construction of the rabbit model. 


\section{Appendix 1: Landscape-Scale Distribution of Rabbit Warrens}

Warren locations recorded in surveys made during unpublished studies owned by Adama Agricultural Solutions Ltd. (reference R-30856, R-30857), undertaken by Rifcon GmbH.

Fig. 5 Locations of rabbit burrow entrances at the study site Würzburg (Bavaria, Germany). a Würzburg_part 'Opferbaum'. b Würzburg — part 'Estenfeld'

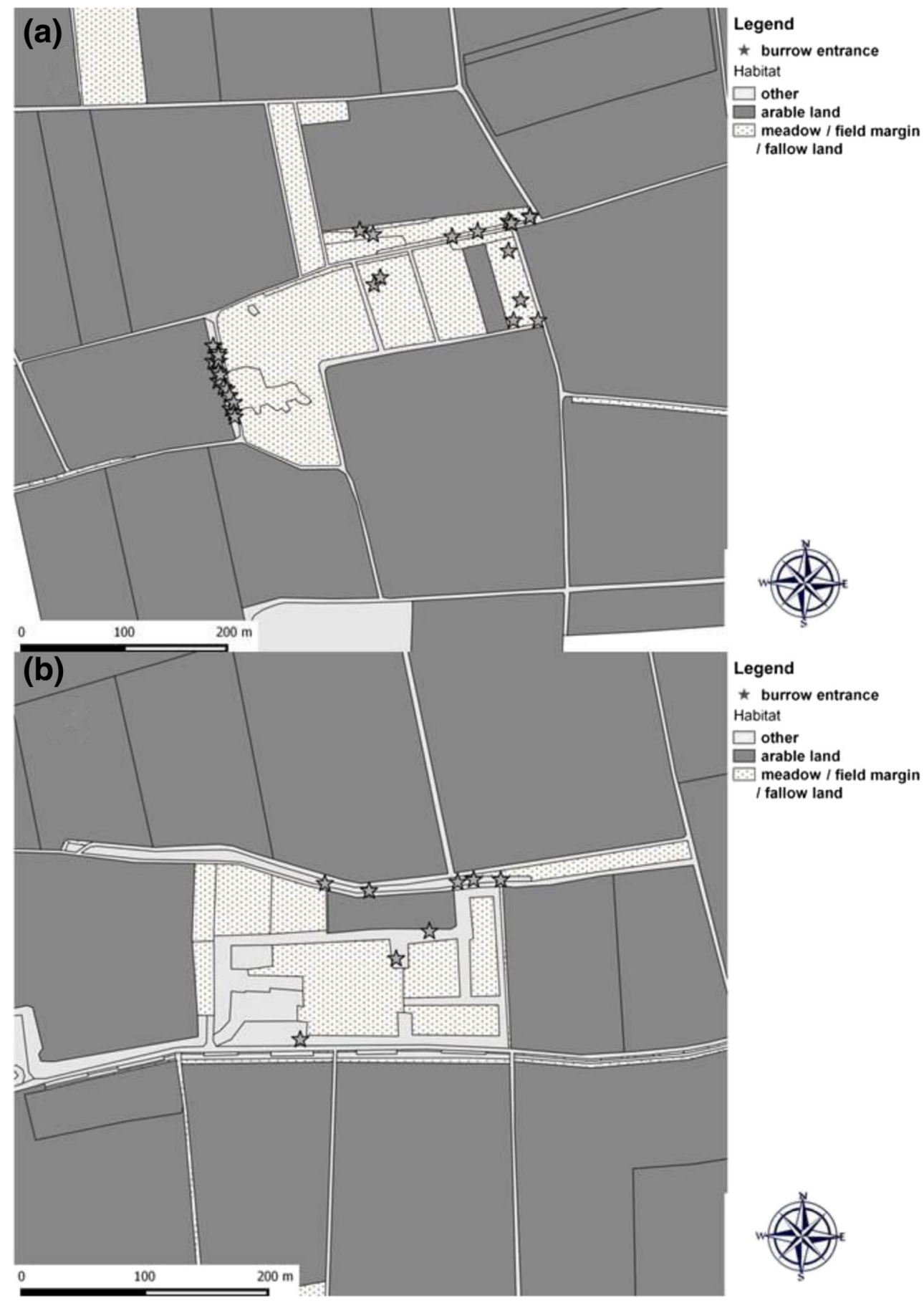


Fig. 6 Locations of rabbit burrow entrances at the study site Paks (Tolna, Hungary). a Pakspart 'valley'. b Paks - part 'pasture'

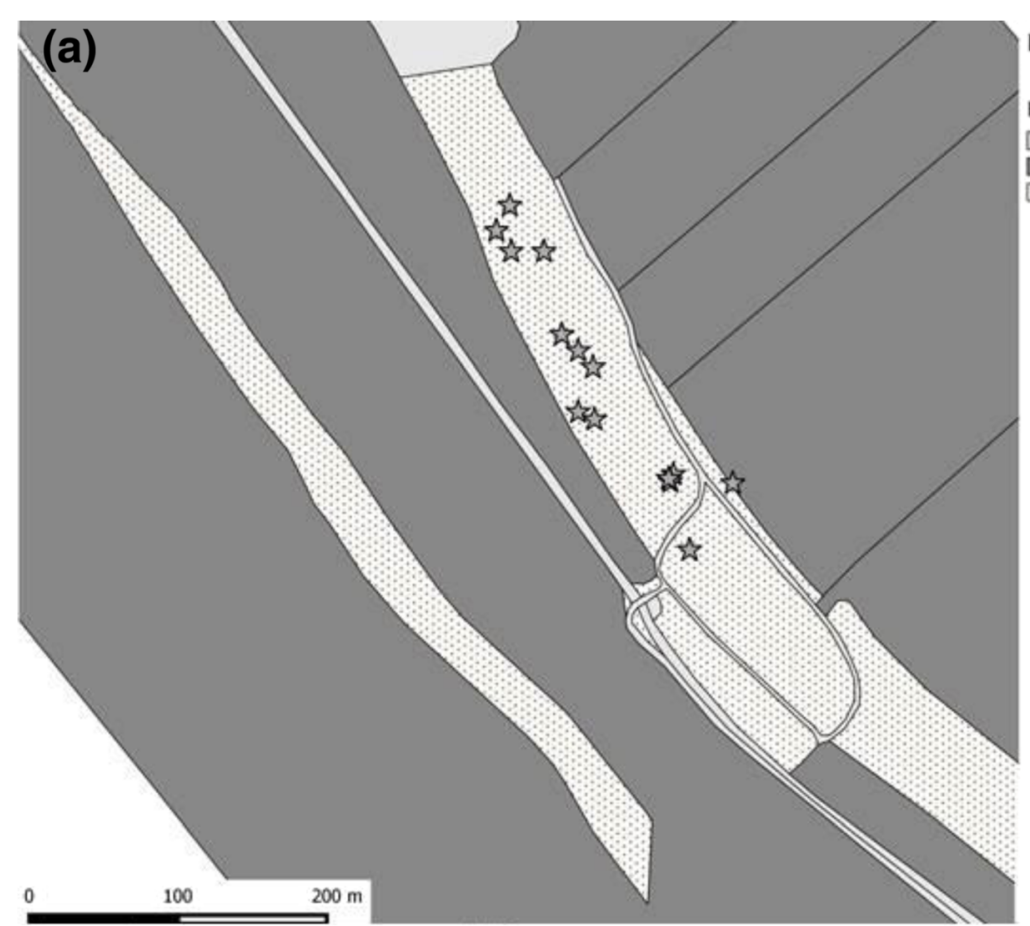

Legend

$\star$ burrow entrance Habitat

$\square$ other

$\square$ arable land

$5-7$ meadow I field margin

I fallow land

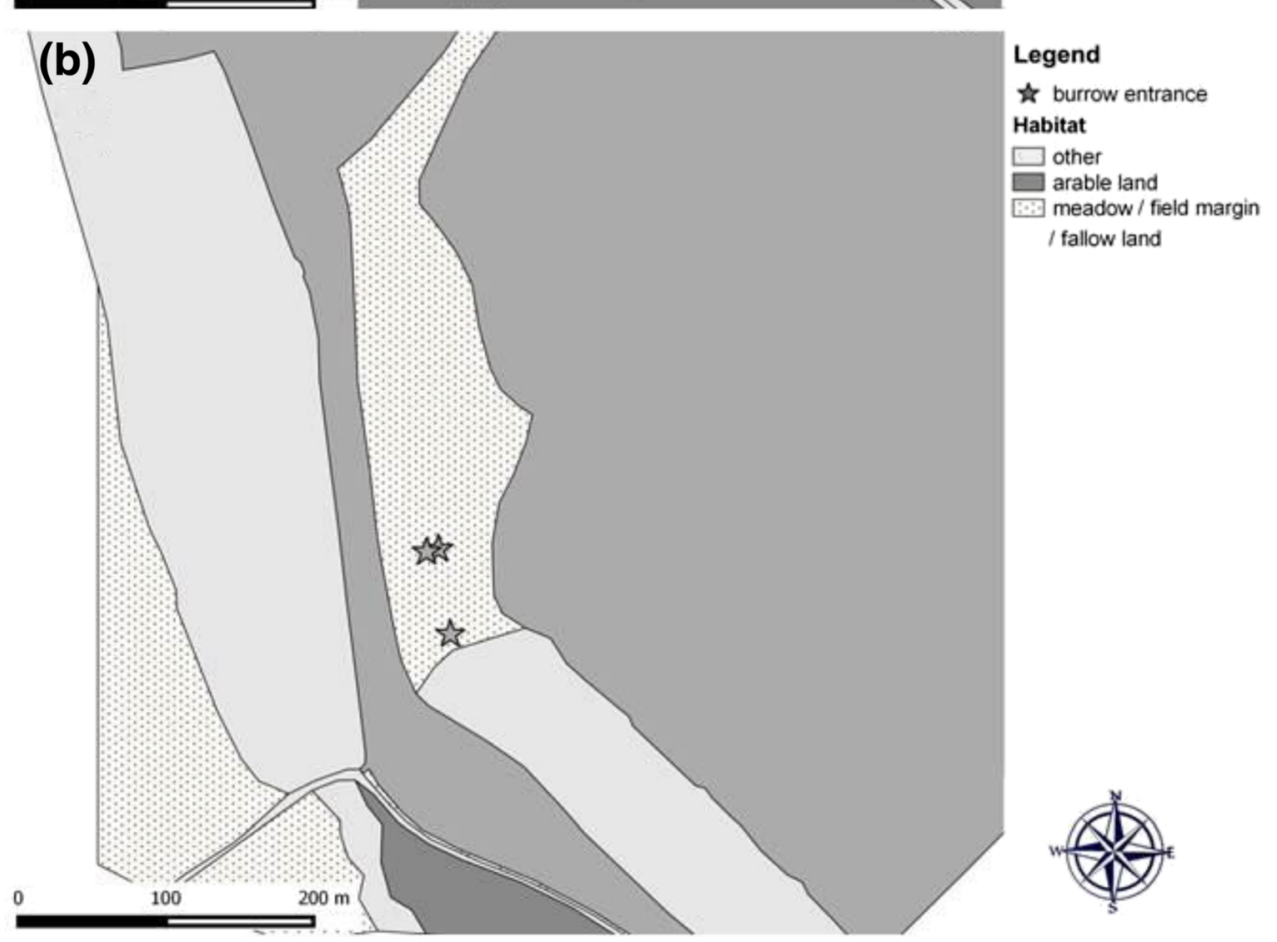


Fig. 7 Location of a rabbit burrow entrances at the study site Paks (Tolna, Hungary). Pakspart 'LPG'

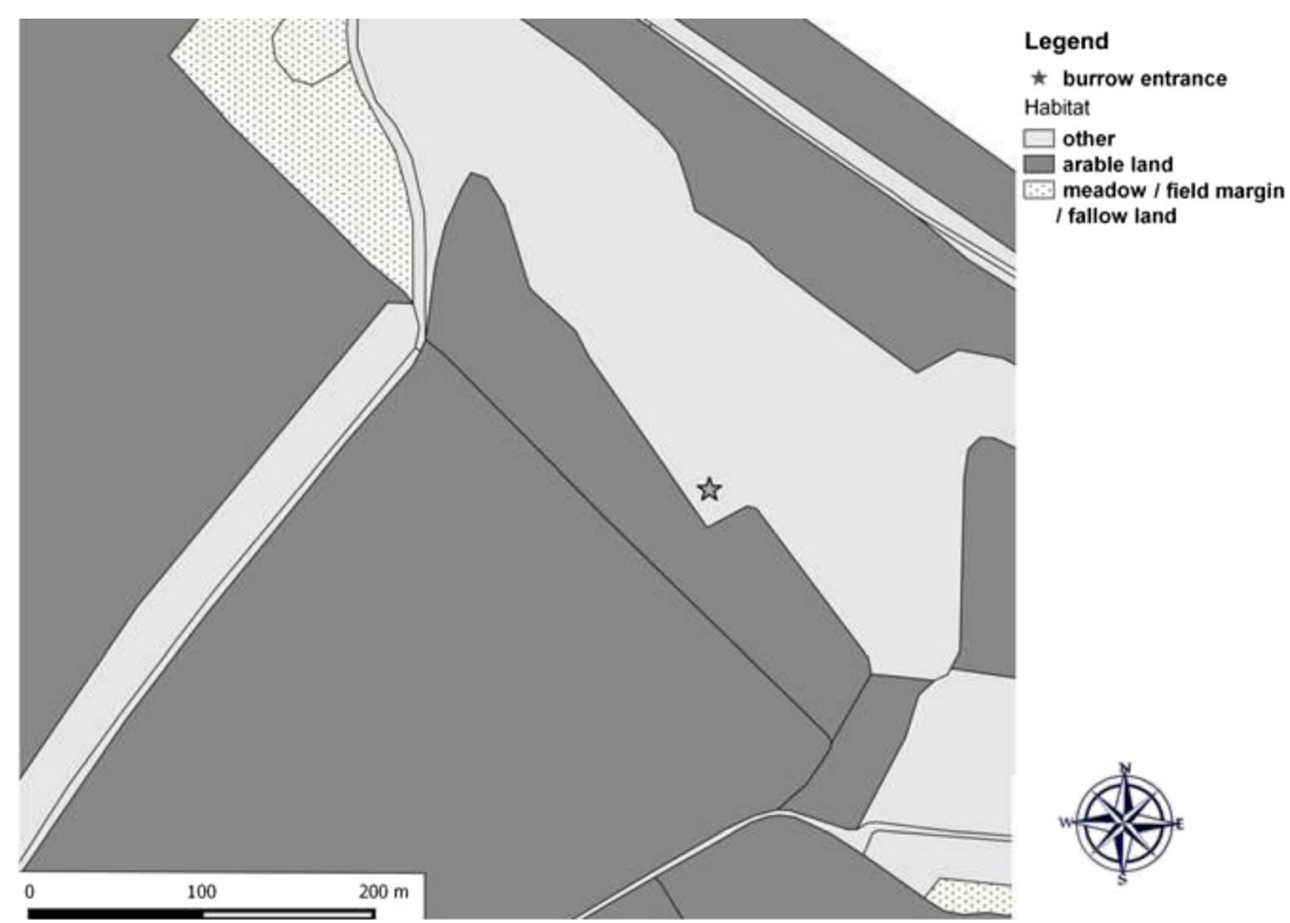


Fig. 8 Locations of rabbit burrow entrances at the study site Albacete (Castile La Mancha, Spain)

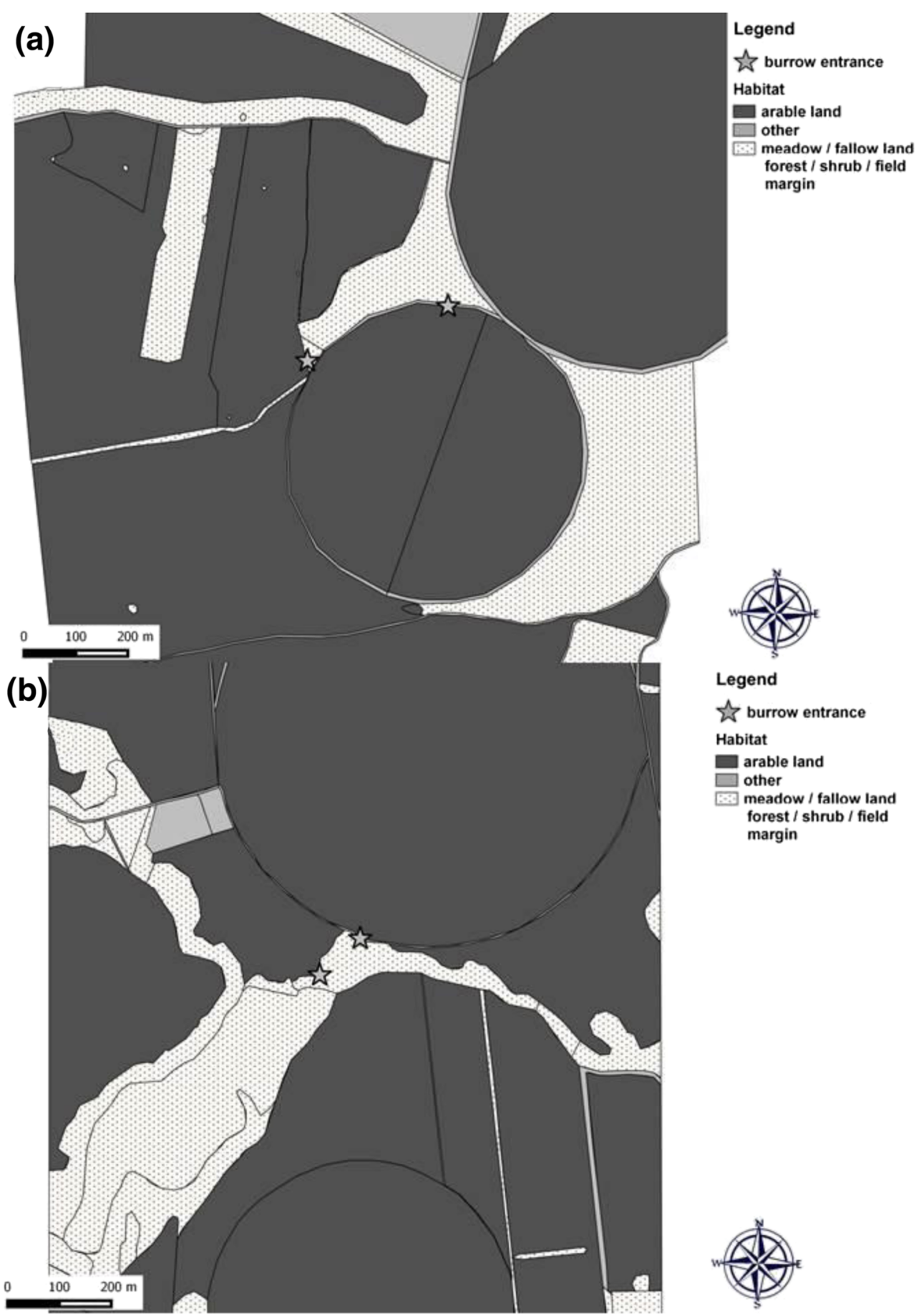


Fig. 9 Locations of rabbit burrow entrances at the study site Albacete (Castile La Mancha, Spain)

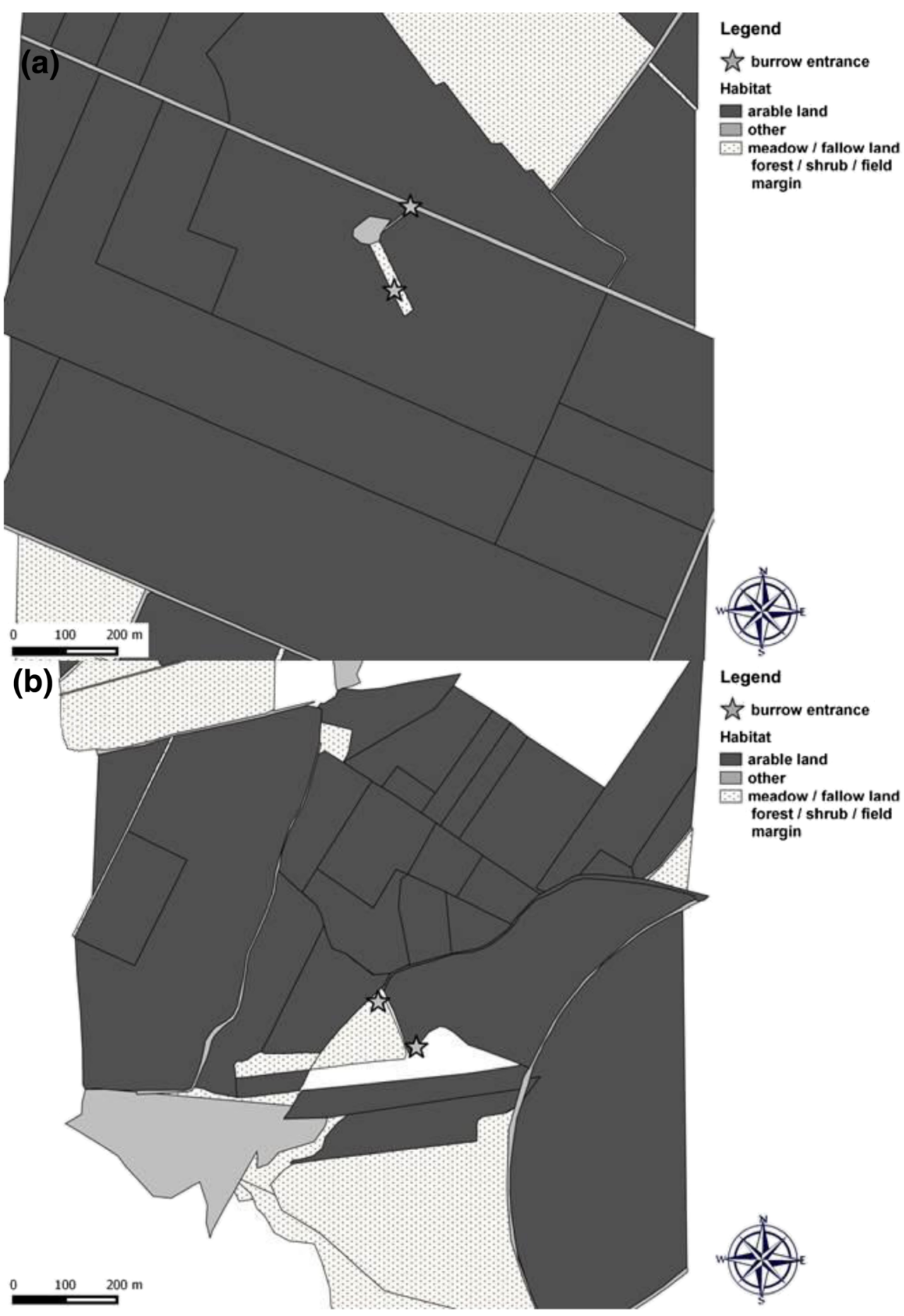


Fig. 10 Locations of rabbit burrow entrances at the study site Albacete (Castile La Mancha, Spain)

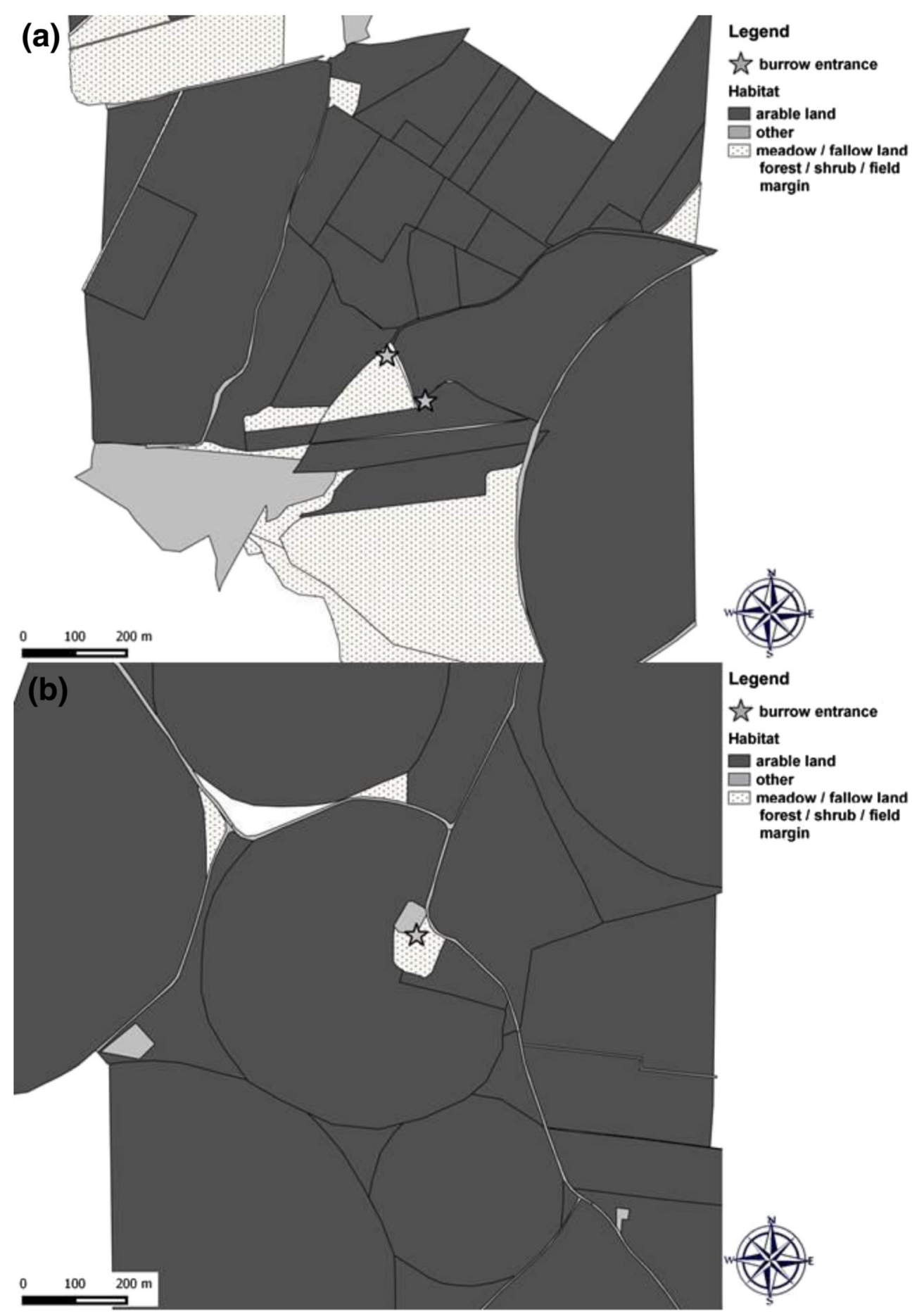


Fig. 11 Locations of rabbit burrow entrances at the study site Albacete (Castile La Mancha, Spain)

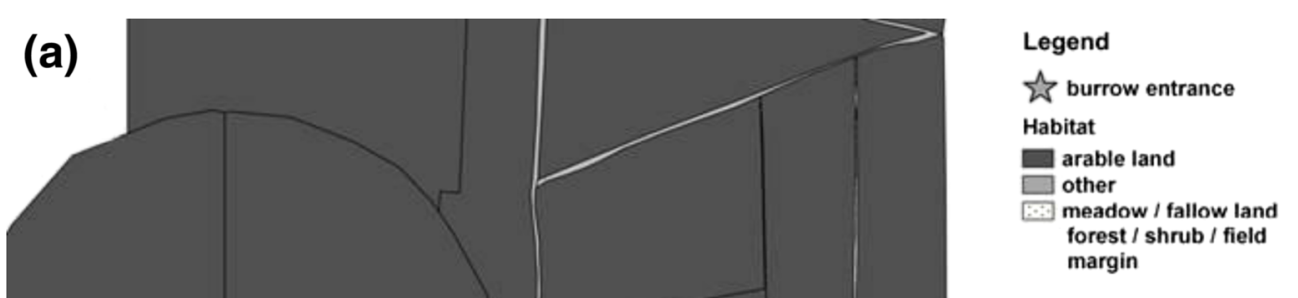

(b)

$0100200 \mathrm{~m}$

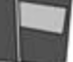

Legend

$\widehat{h}$ burrow entrance

Habitat

arable land

$\square$ other

meadow / fallow land

forest / shrub / field

margin
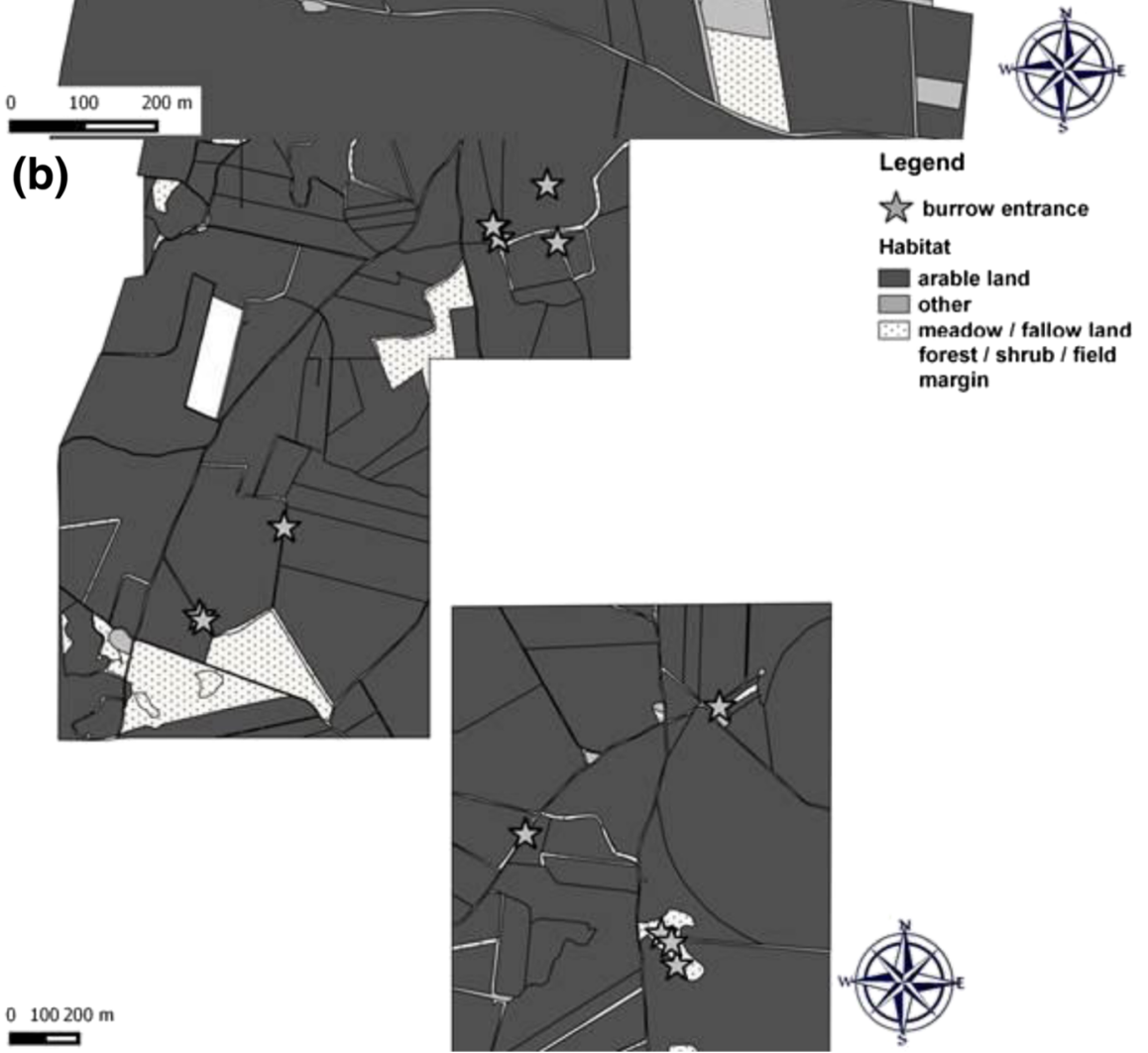
Fig. 12 Locations of rabbit burrow entrances at the study site Albacete (Castile La Mancha, Spain)
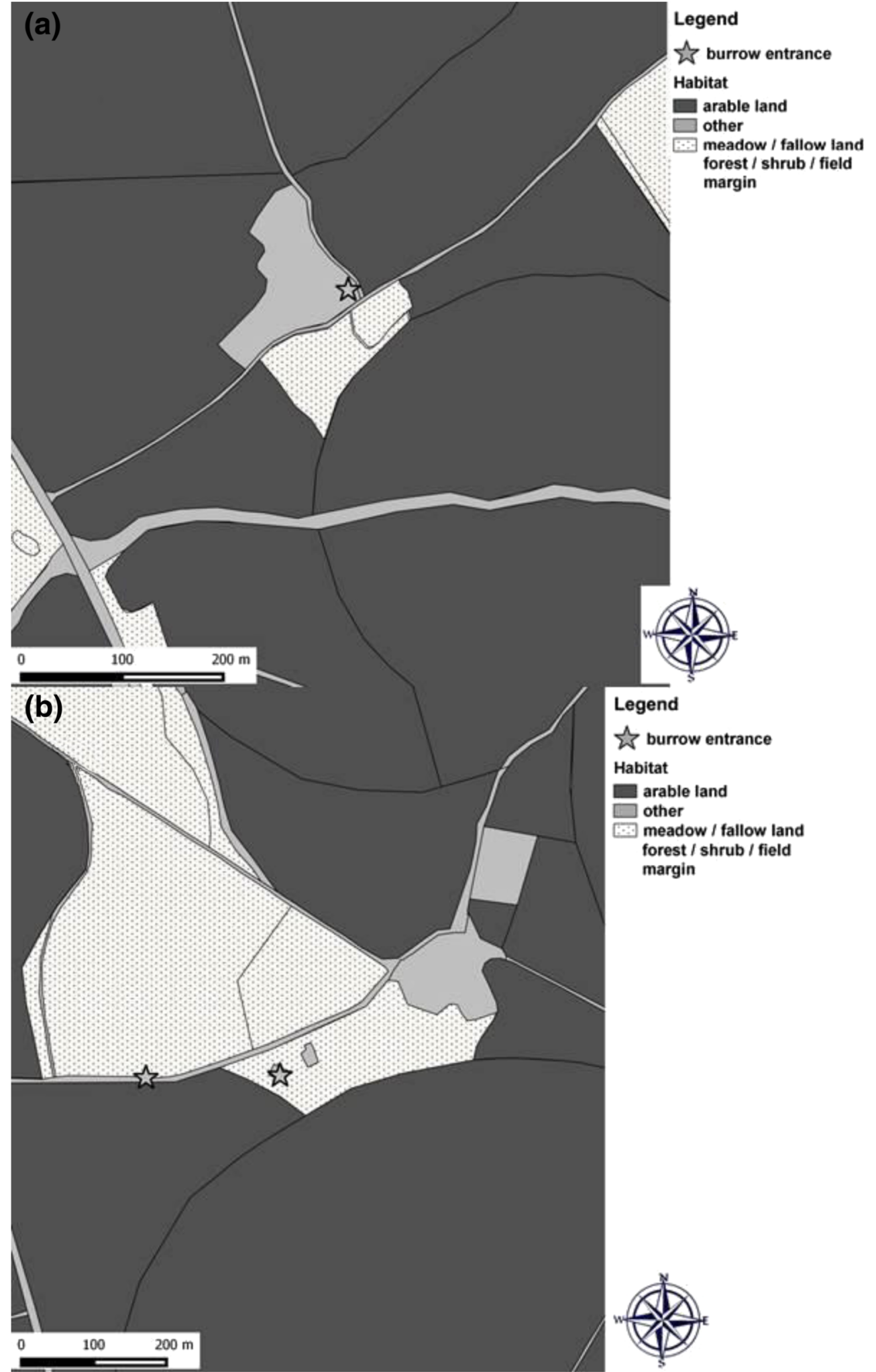

$\hat{2}$ burrow entrance

Habitat

$\square$ arable land

$\square$ other

meadow / fallow land

forest / shrub / field

margin 
Fig. 13 Locations of rabbit burrow entrances at the study site Seville (Andalucia, Spain)

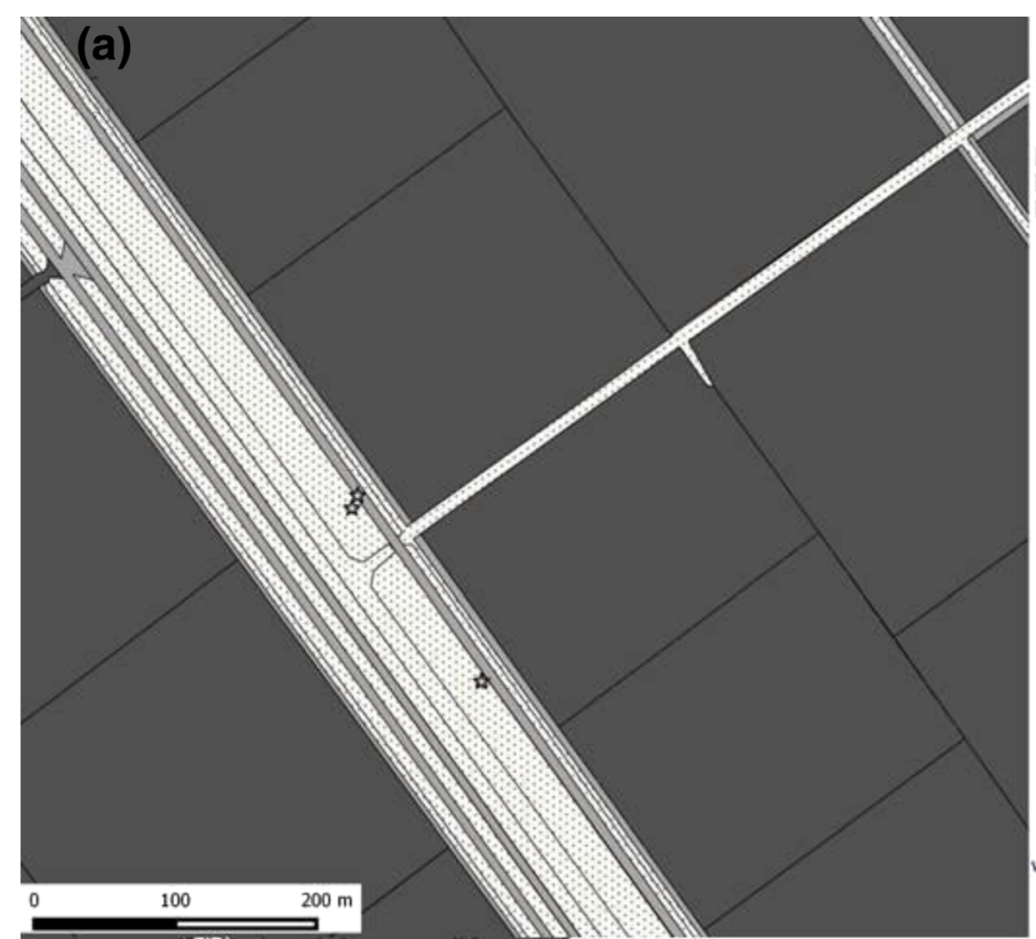

Legend

s burrow entrance Habitat

$\square$ arable land

$\square$ other

meadow / fallow land forest / shrub / field margin

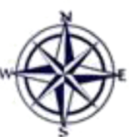

Legend

岤 burrow entrance Habitat $\square$ arable land

$\square$ other

meadow / fallow land forest / shrub / field margin

$0100200 \mathrm{~m}$
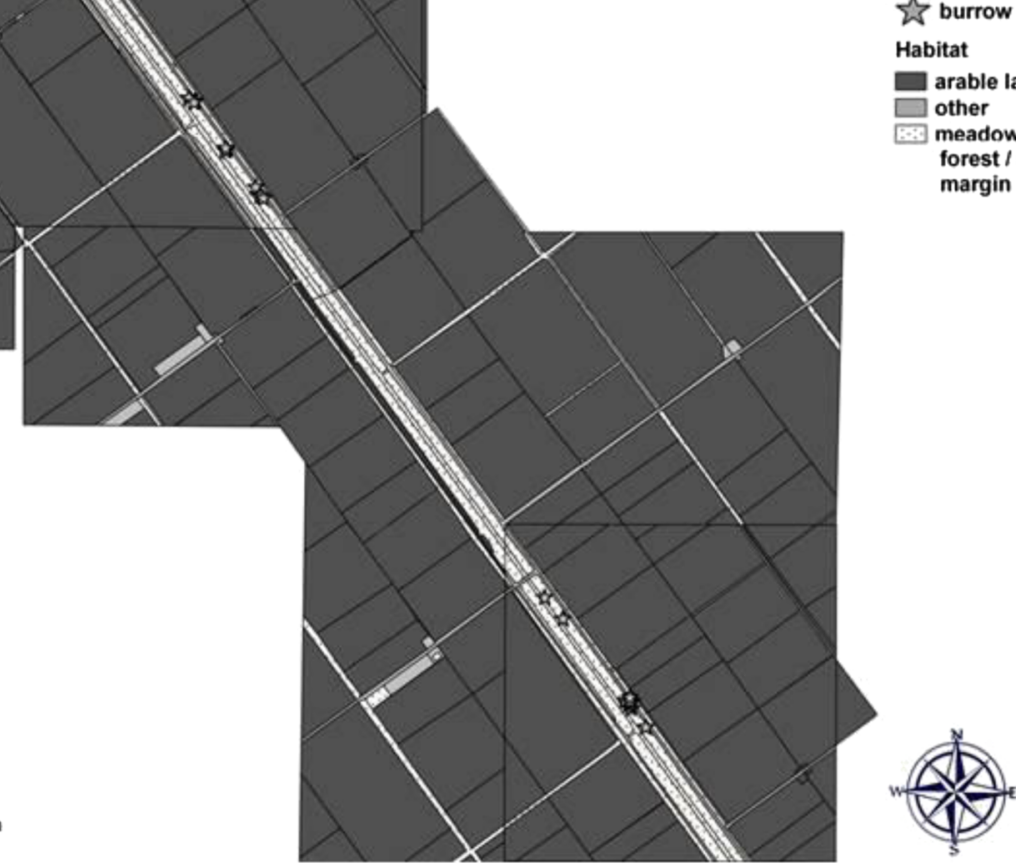
Fig. 14 Locations of rabbit burrow entrances at the study site Seville (Andalucia, Spain)

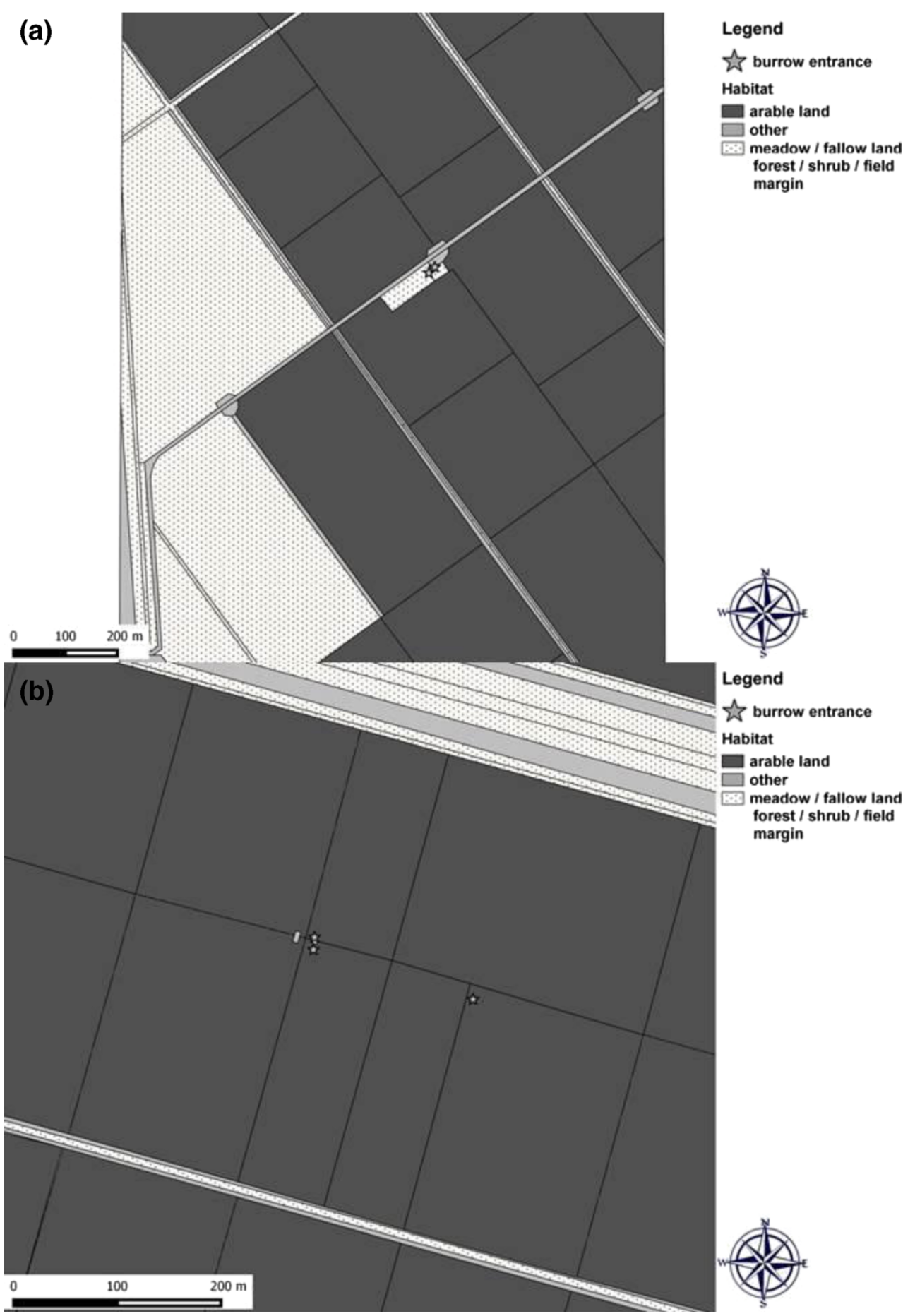


Fig. 15 Locations of rabbit burrow entrances at the study site Seville (Andalucia, Spain)

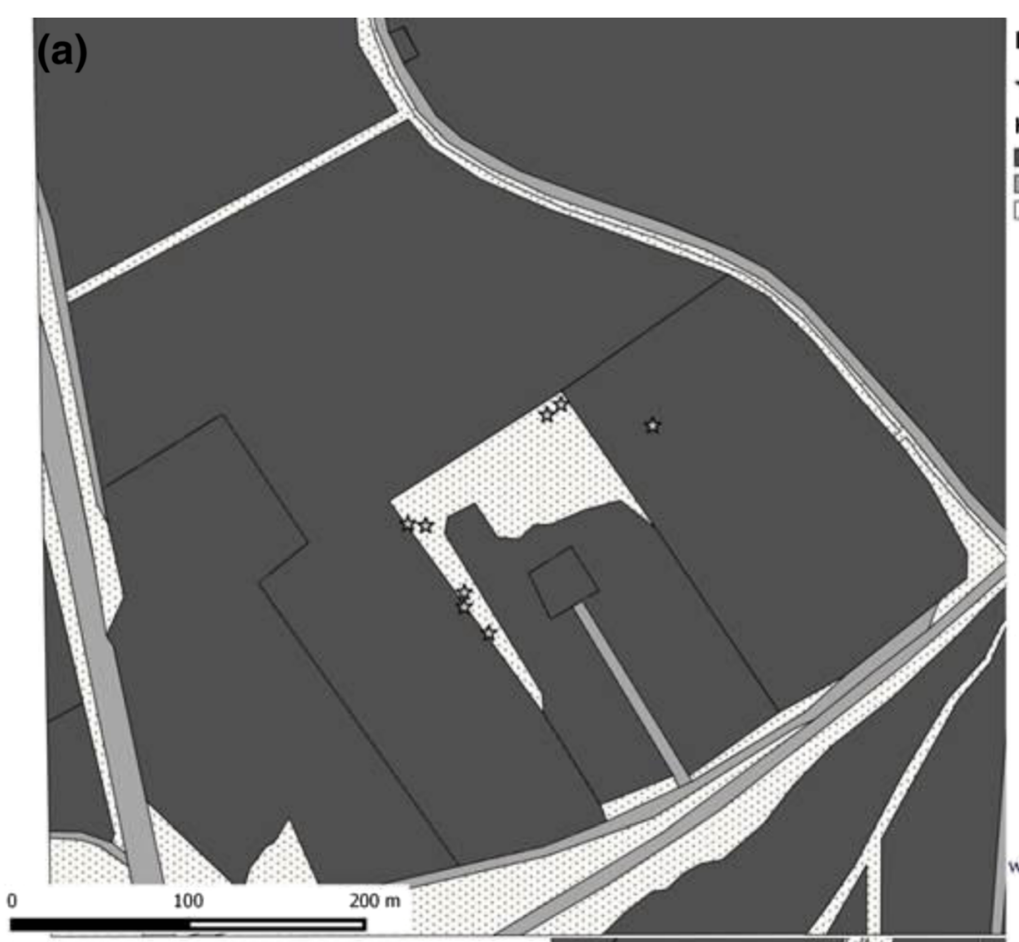

Legend

burrow entrance

Habitat

$\square$ arable land

other

meadow / fallow land

forest / shrub / field

margin

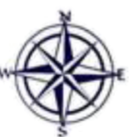

Legend

(b)

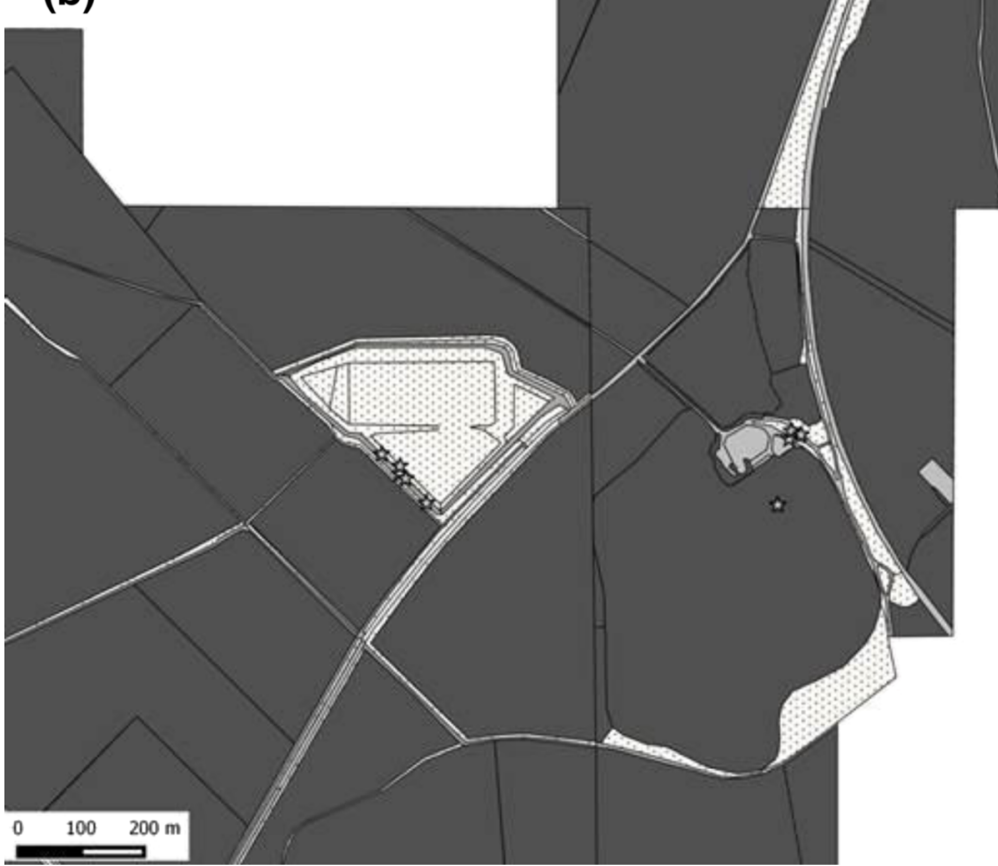

th burrow entrance

Habitat

$\square$ arable land

$\square$ other

meadow / fallow land forest / shrub / field margin

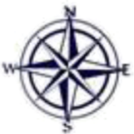




\section{Appendix 2: Sensitivity Analysis}

Each parameter was varied by $\pm 25,50$ and $75 \%$ of its value in Table 2 of the main text. The proportion change in 13 output patterns for the 17 POM patterns used for fitting is shown graphically below together with short notes on what the parameter does in the model. Please also see the ODdox documentation for the model for more details on parameter usage.

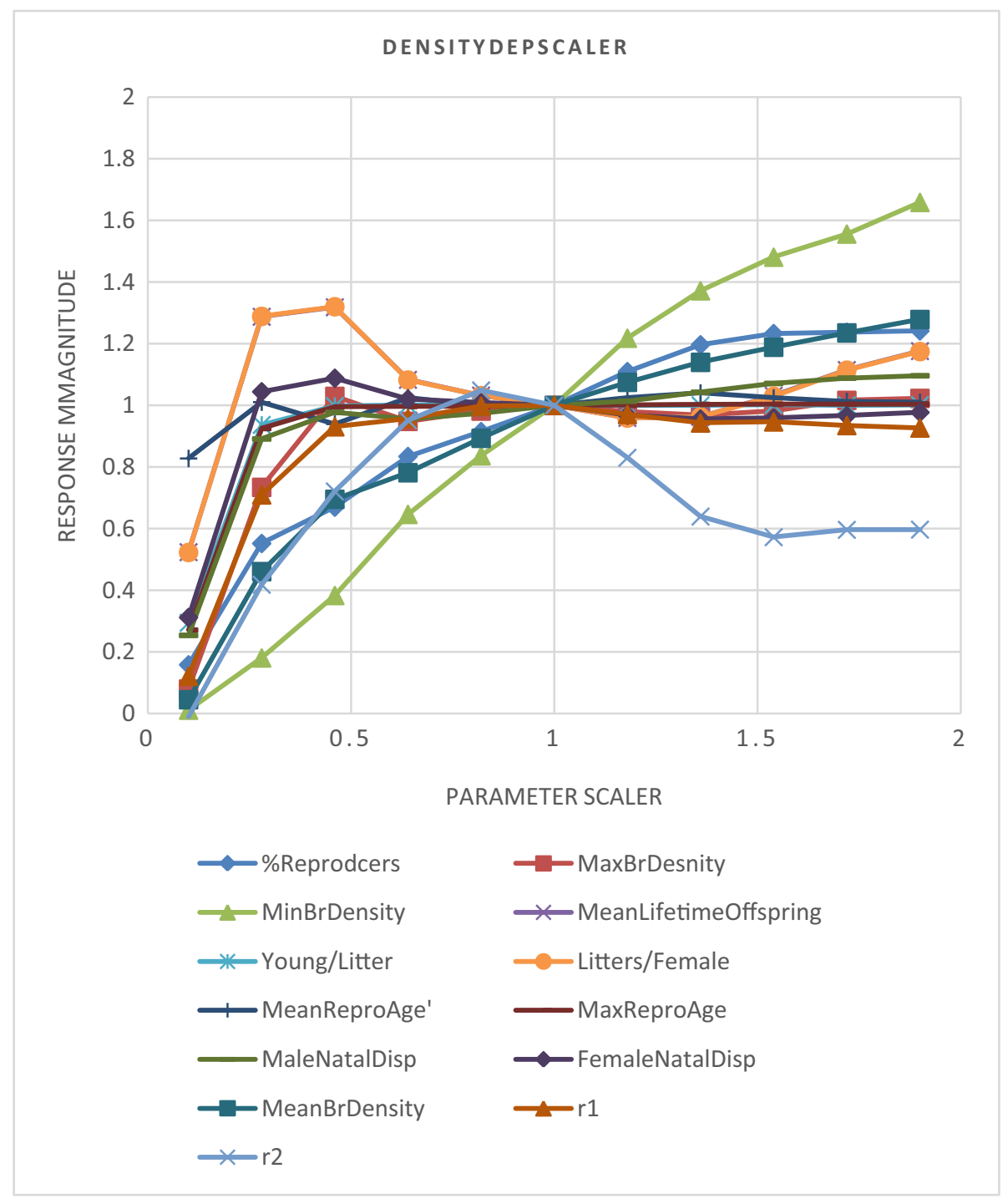

DENDEPSCALER is a constant used to scale the nominal carrying capacity with the effect of increasing or decreasing the effect of density dependent forage and mortality calculations. 


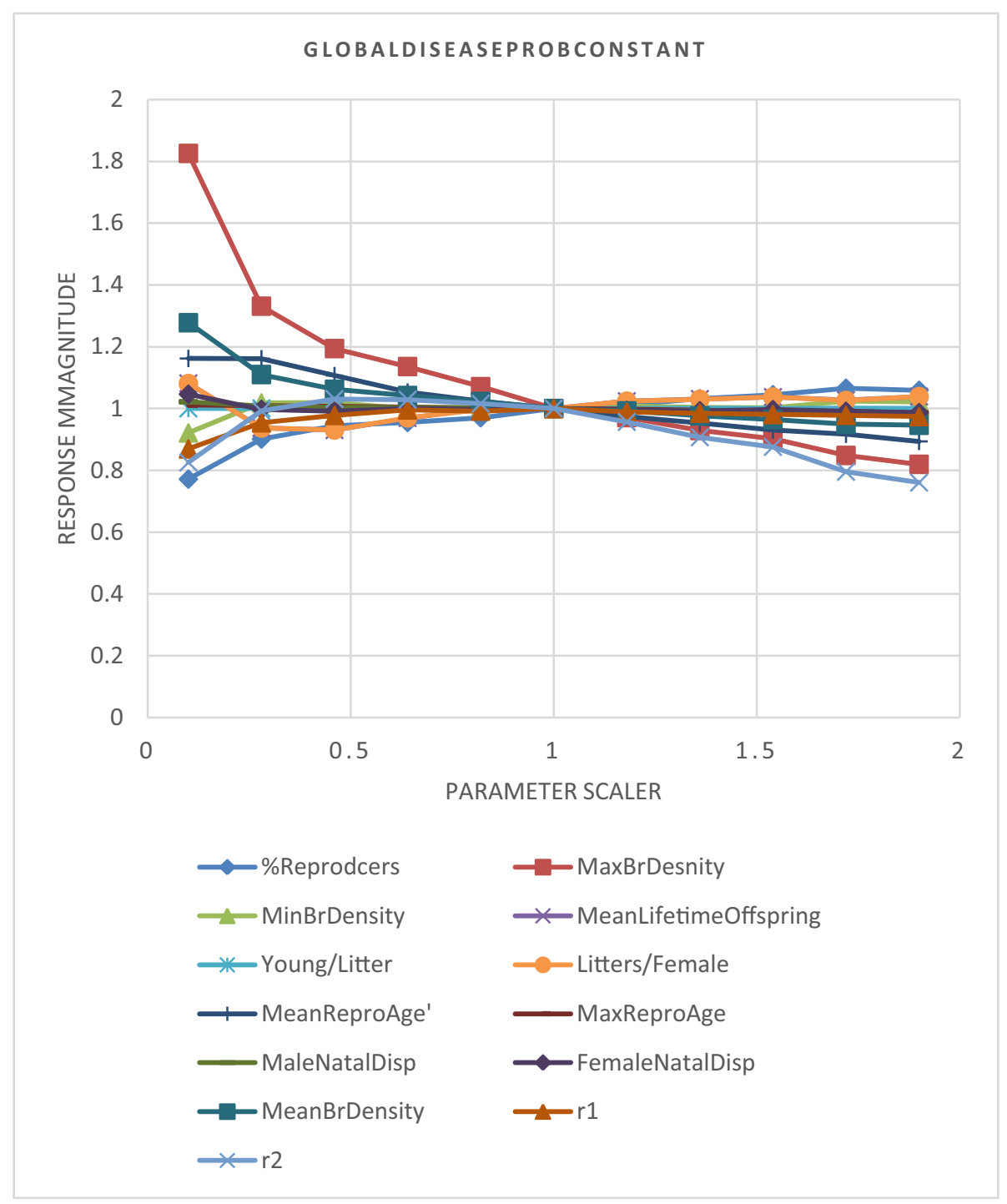

GLOBALDISEASEPROBCONSTANT is the constant that determines probability of globally calculated disease mortality together with the global rabbit population size. The disease chance is based on global density: chance $=$ (totalrabbits/no warren) $\times$ (totalrabbits/no warren $\times$ GLOBALDISEASEPROBCONSTANT. This is then modified by local density: chance $=$ prob_density $\times$ CarryingCapacityRatio2. where carrying capacity ratio is the mean number of female rabbits over 300 days divided by the carrying capacity ratio (CarryingCapacity $R / 2$ ) of each warren within the current warren network. CarryingCapacity $R$ is the number of burrows possible in a warren multiplied by DENDEPSCALER. 


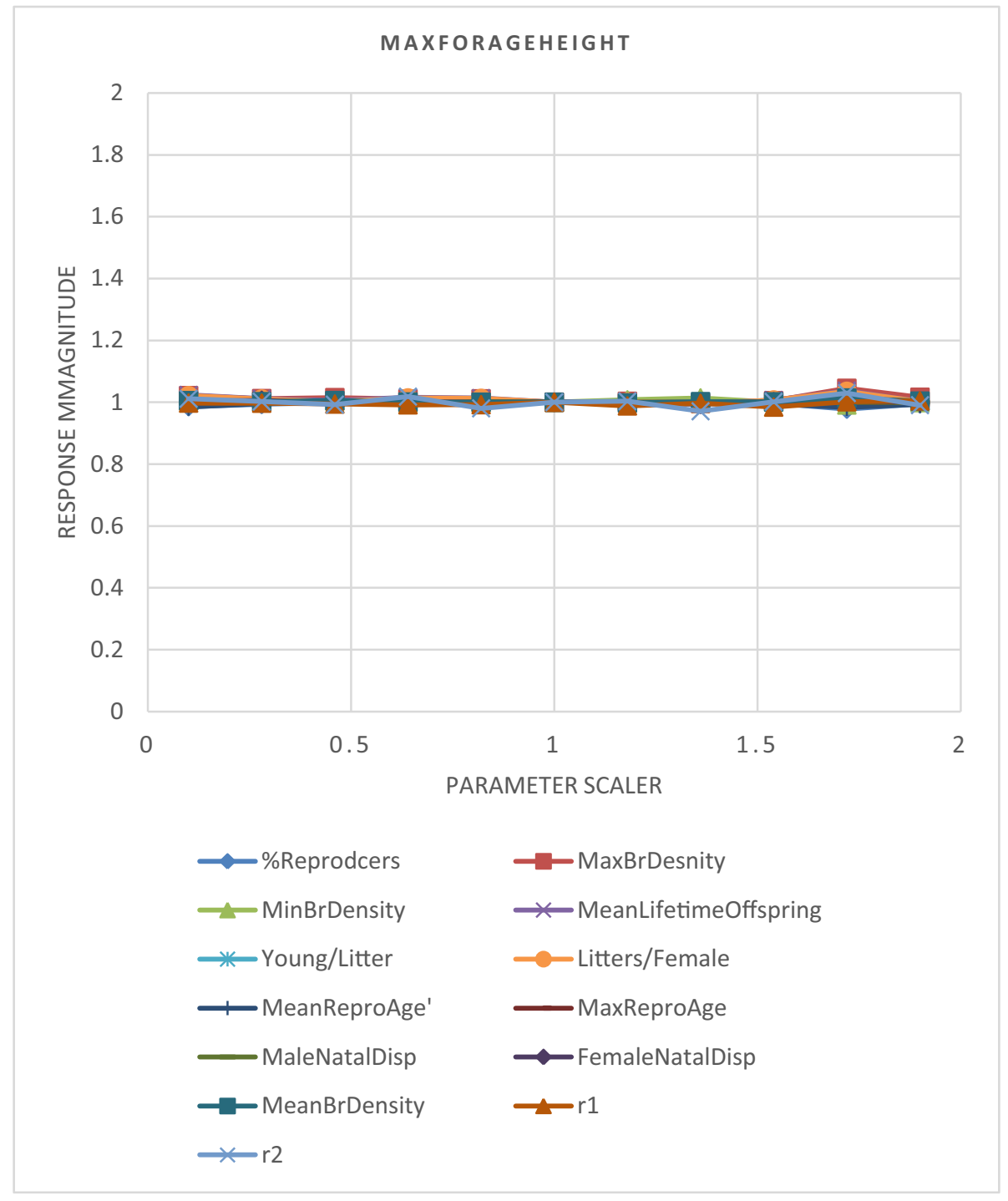

MAXFORAGEHEIGHT is the height at which efficient foraging is assumed to be impossible. This primarily affects the carrying capacity of the warren at any point in time where forage area below this value is limiting. This is used to calculate the ratio between forage area and available forage. If available/total is less than CarryingCapacityRatio2, then the available forage is divided by CarryingCapacityRatio 2 and this is used in litter absorption calculations. 


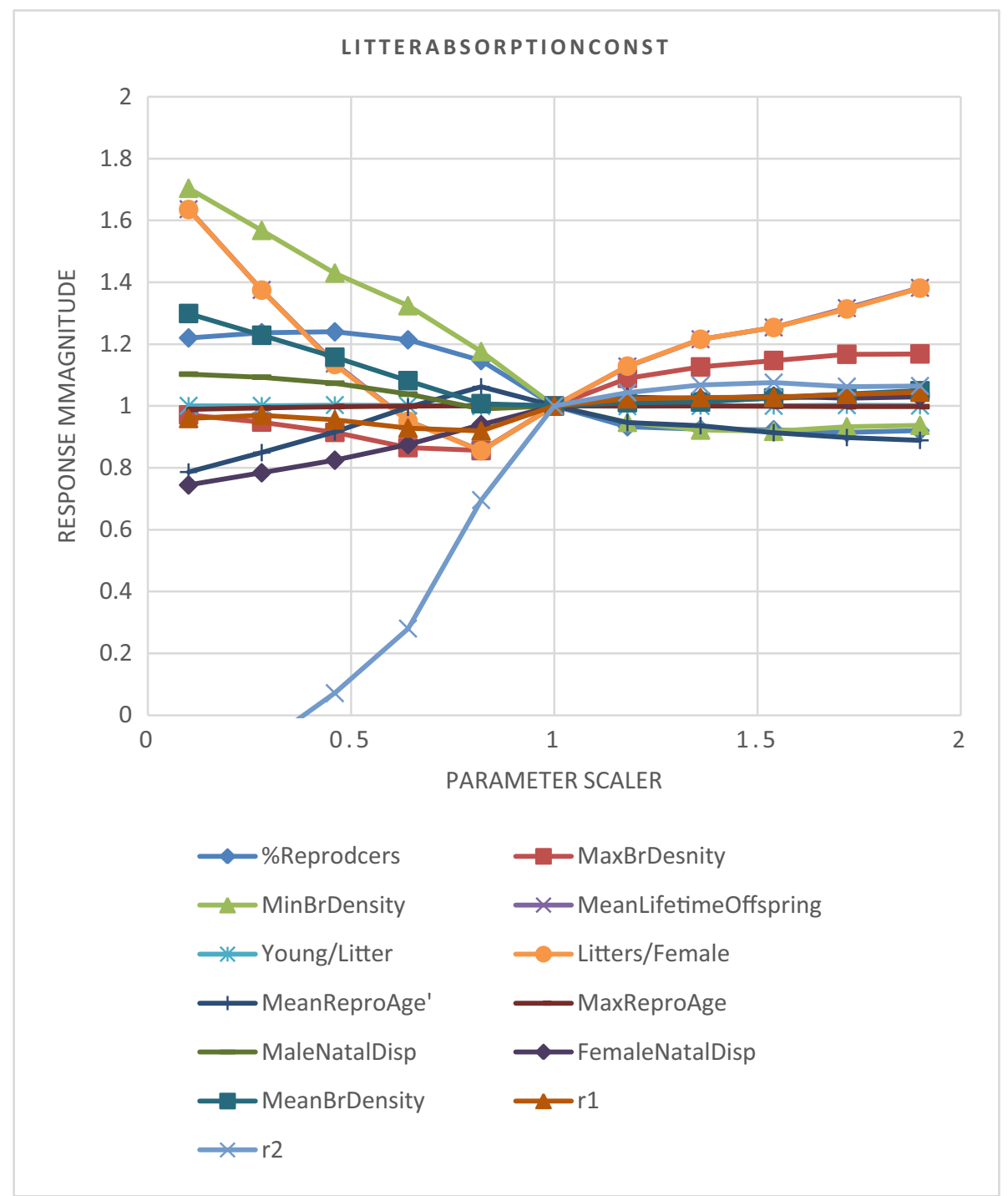

LITTERABSORPTIONCONST is the power to which the relationship between local warren density and the probability of reabsorption of a litter was raised: chance $=$ LITTERABSORPTIONCONST $\times(1.0-\text { available forage })^{\text {LITTERABSORPTIONCONST }}$. where available forage is the ratio between available area and potential forage modified by the ratio of current-carrying capacity to number of rabbits in the warren. 


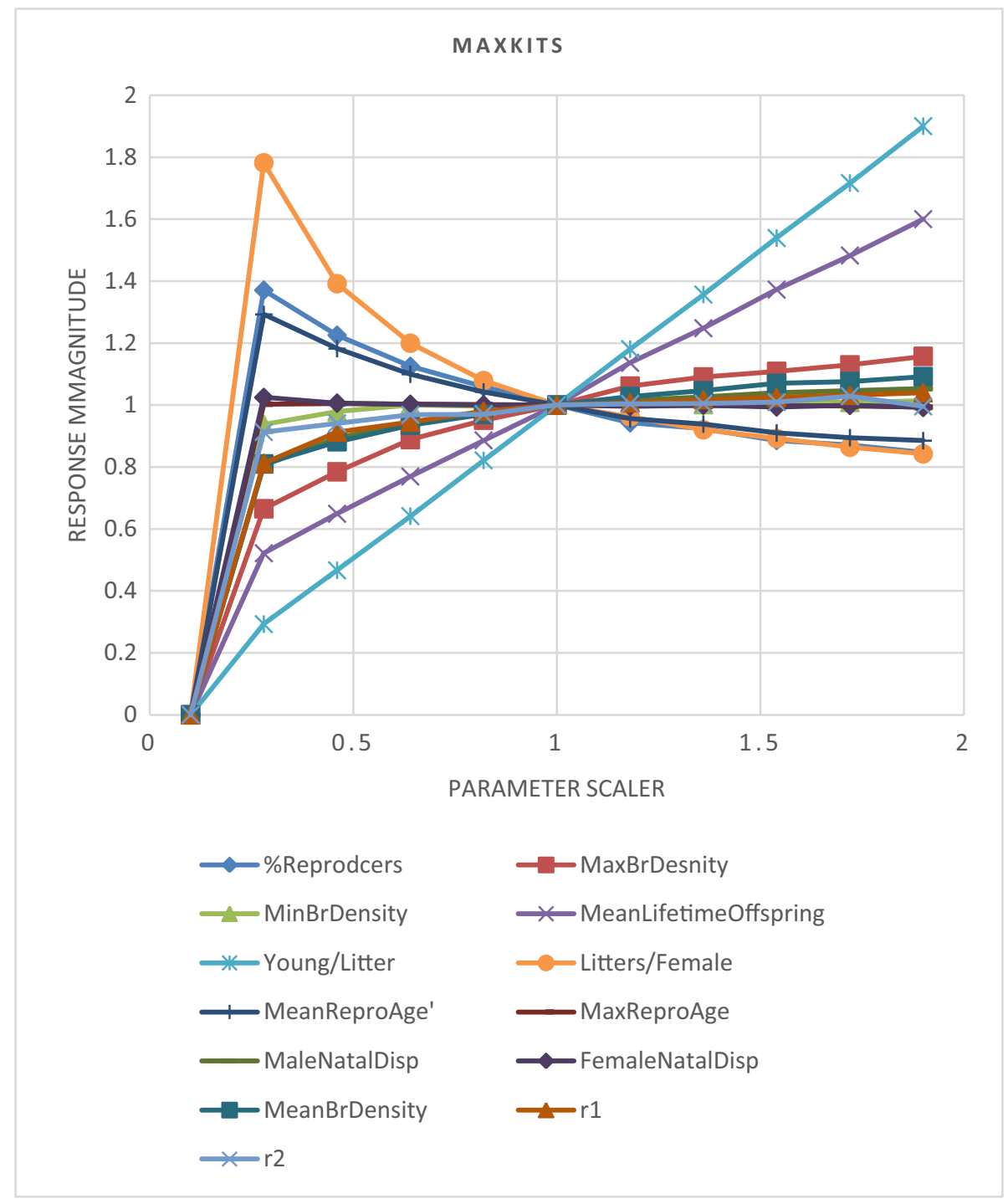

MAXKITS is the maximum number of young per litter. The number of young per litter is evenly distributed between this value and 1. 


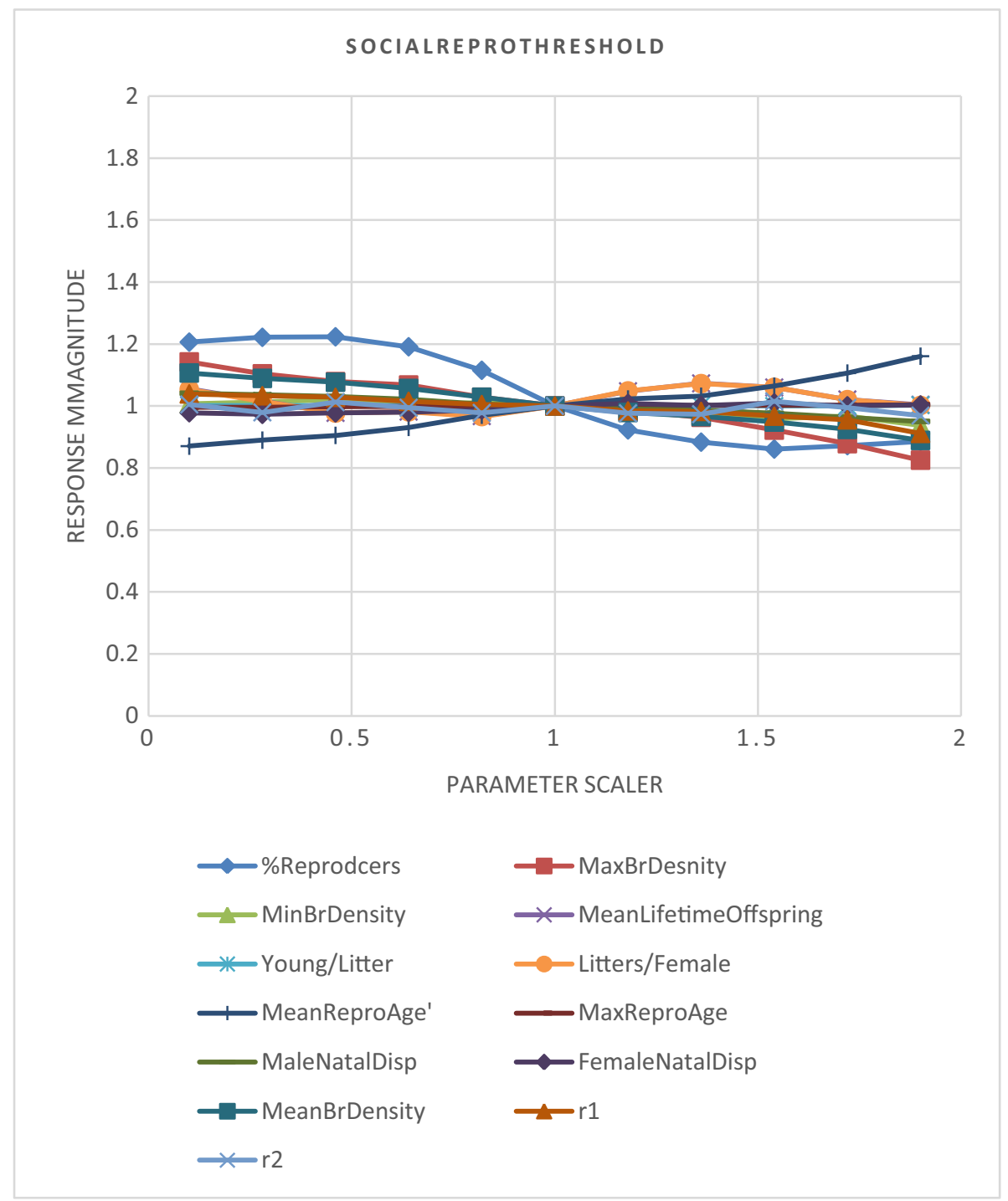

SOCIALREPROTHRESHOLD is a constant used to determine the probability of reproduction of rabbits who have the social status sub-dominant. Higher values decrease the chance of reproduction. 


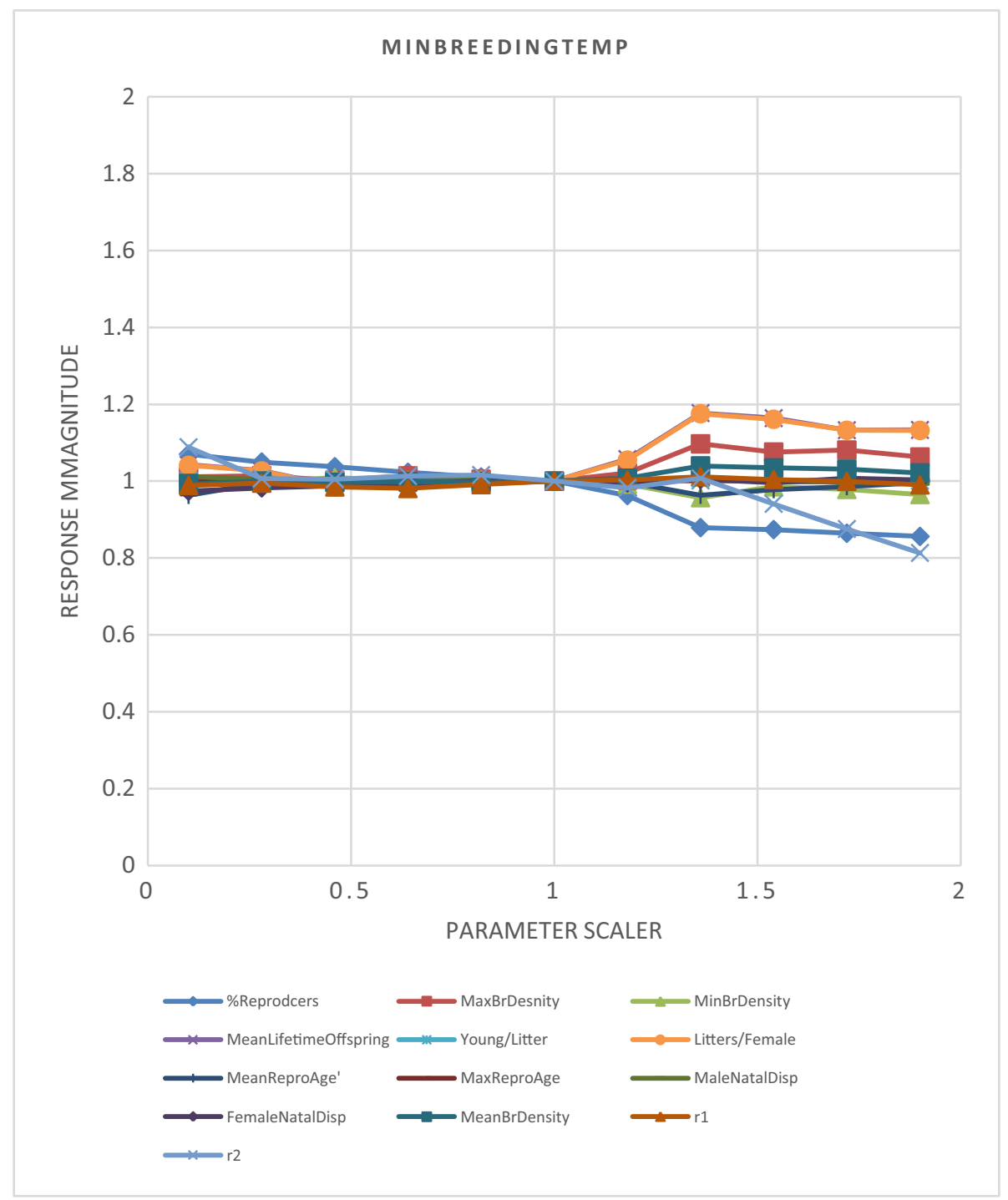

MINBREEDINGTEMP is the minimum summed temperature over 60 days before a rabbit can start breeding, or at which it will stop breeding. 


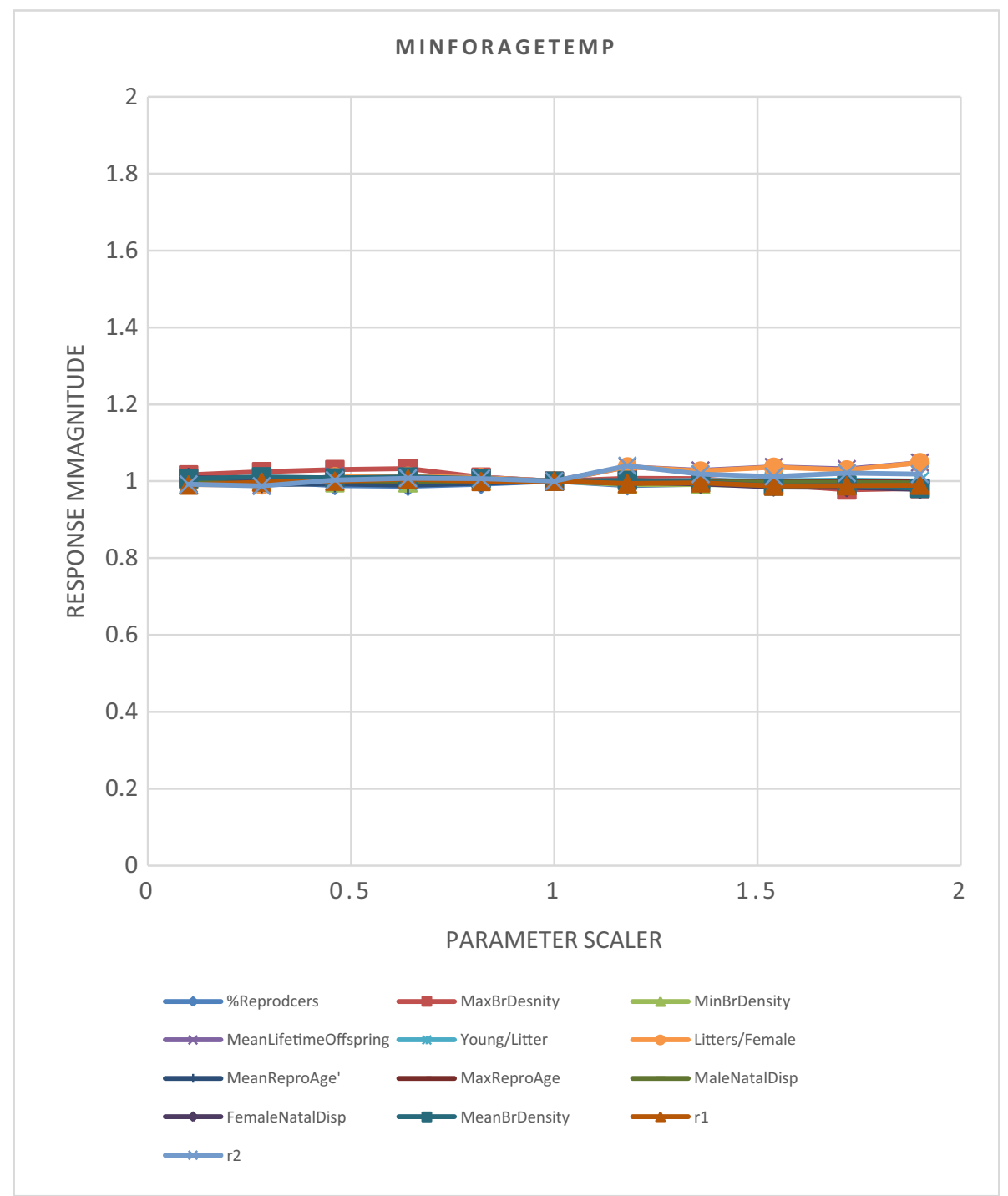

MINFORAGETEMP is minimum temperature in degrees Celsius that set as the threshold for efficient foraging to occur. This primarily affects juvenile growth and forage-related mortality. 


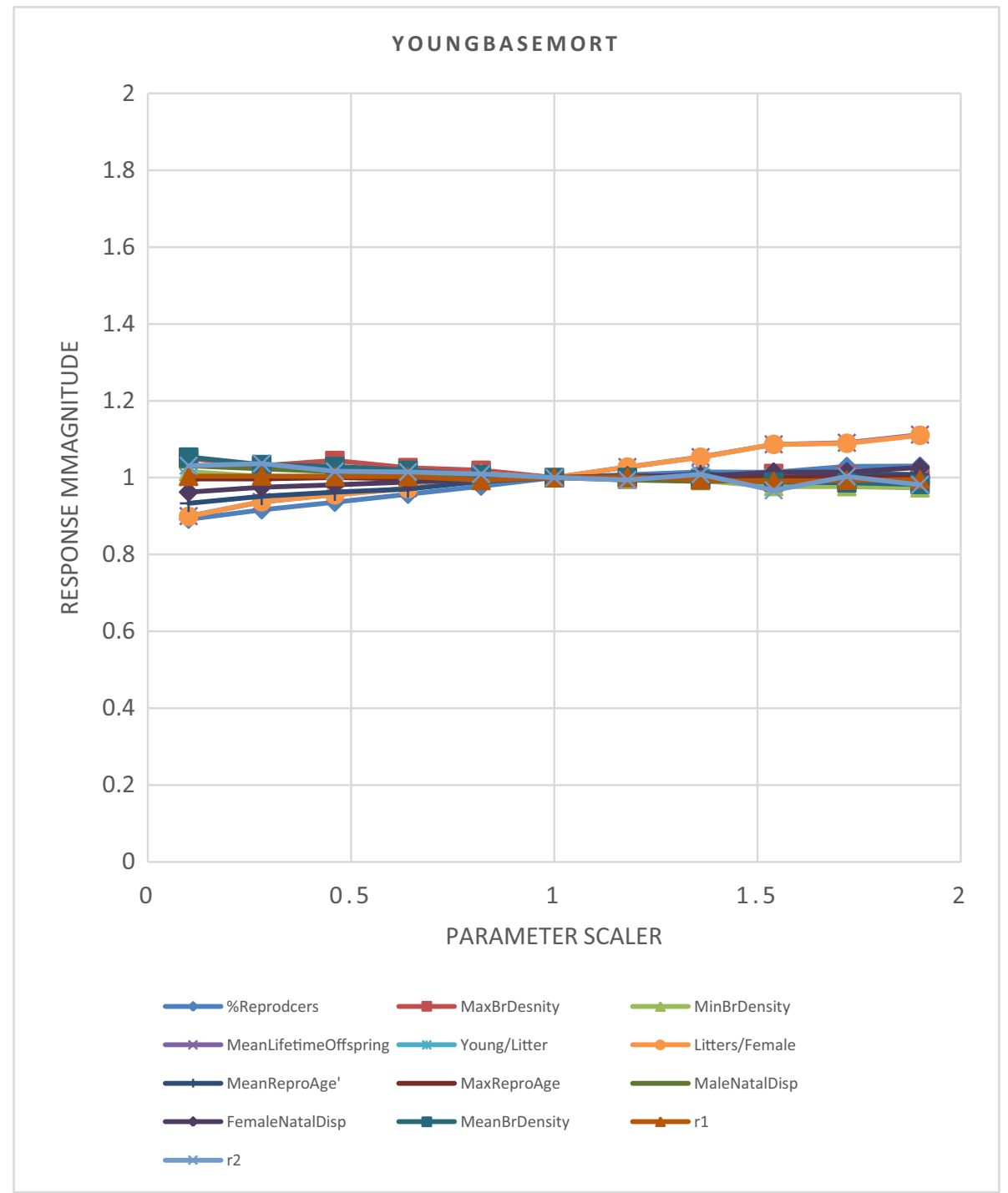

YOUNGBASEMORT is the daily probability of mortality for the Rabbit_Young stage before any other external mortality or mortality multipliers are applied. 


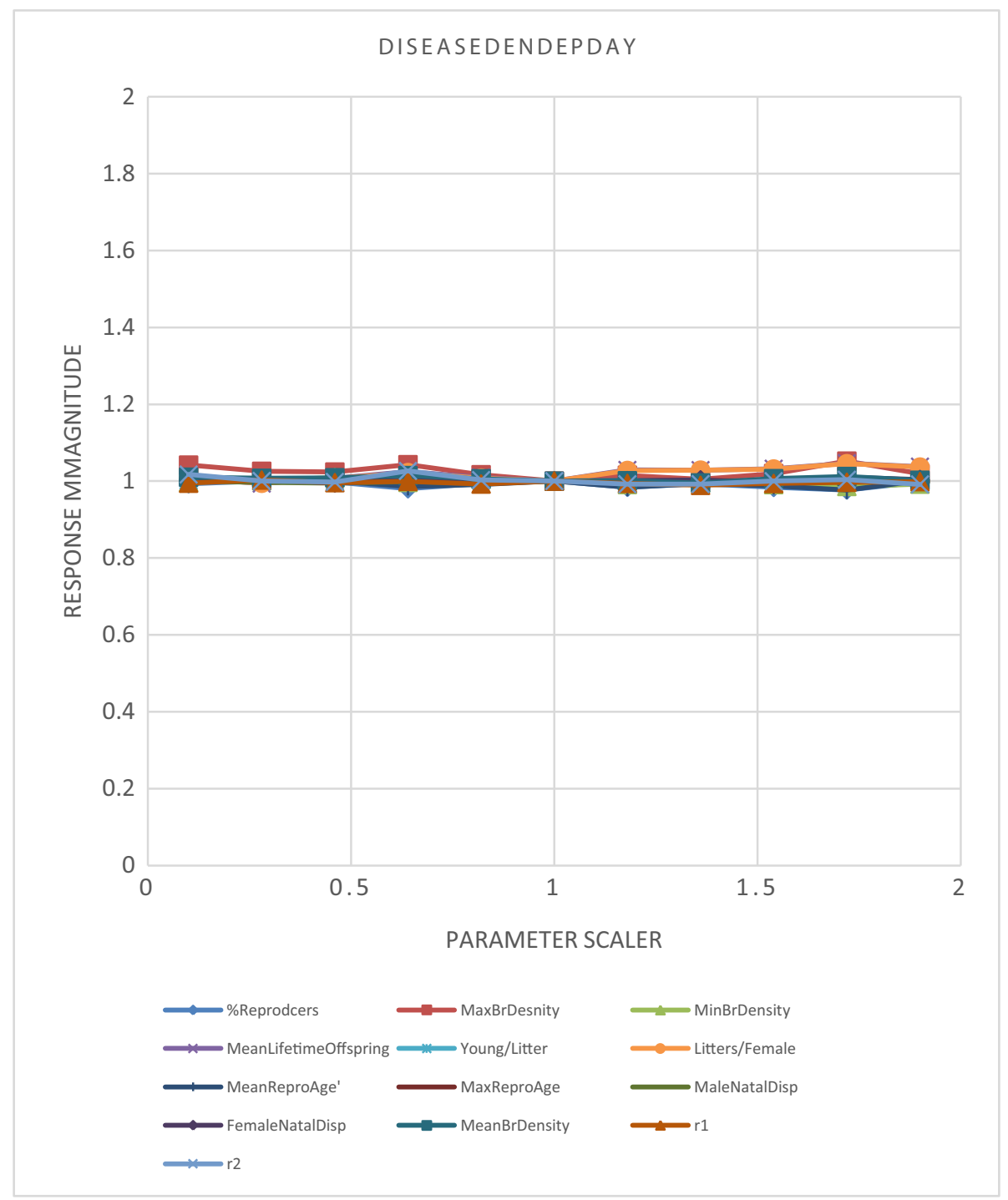

DISEASEDENDEPDAY is the day in the year that the density-dependent disease calculation is carried out. 


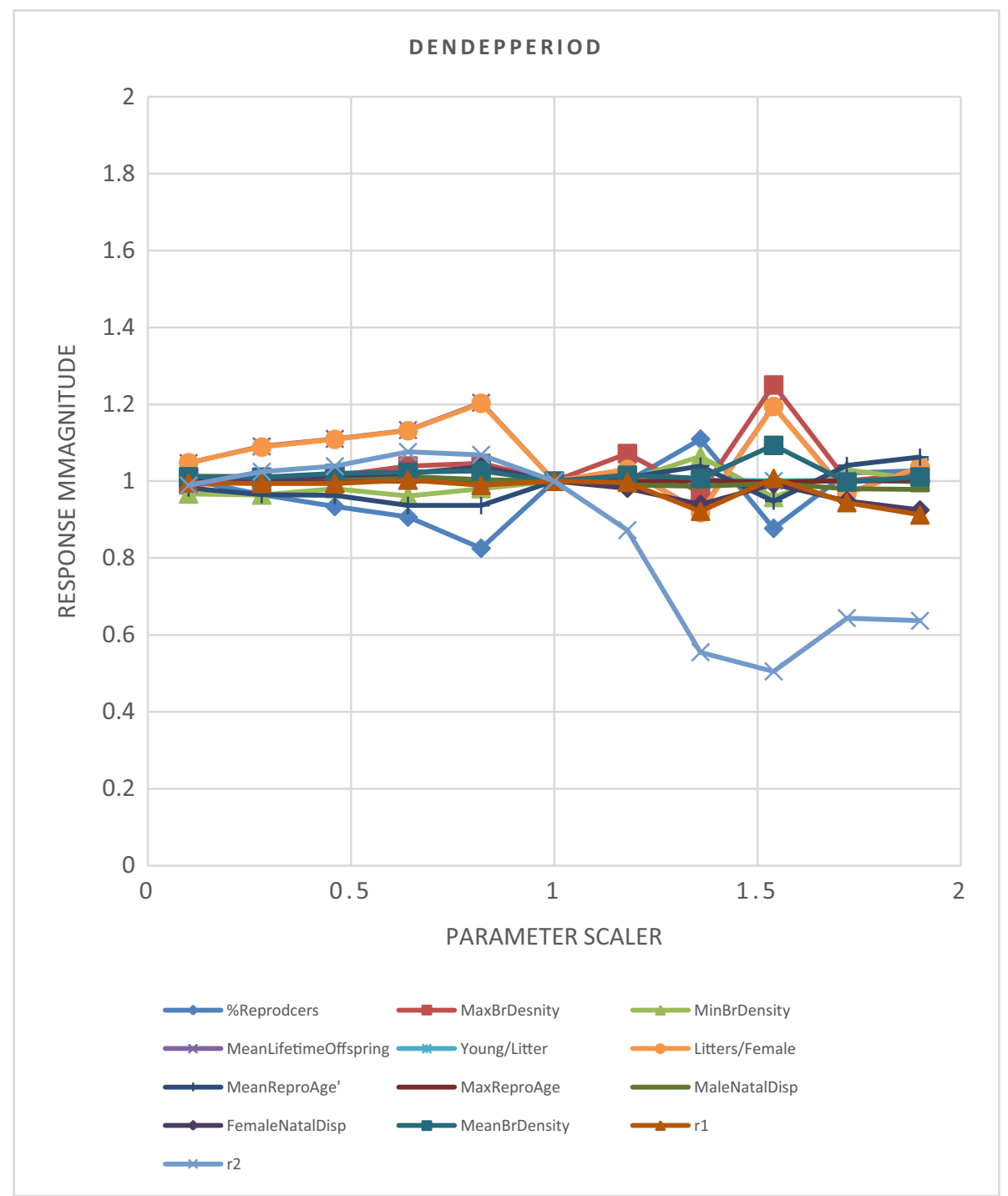

DENDEPPERIOD is the periodicity in days between the recalculation of density-dependent disease probabilities and densityrelated reductions in foraging. 


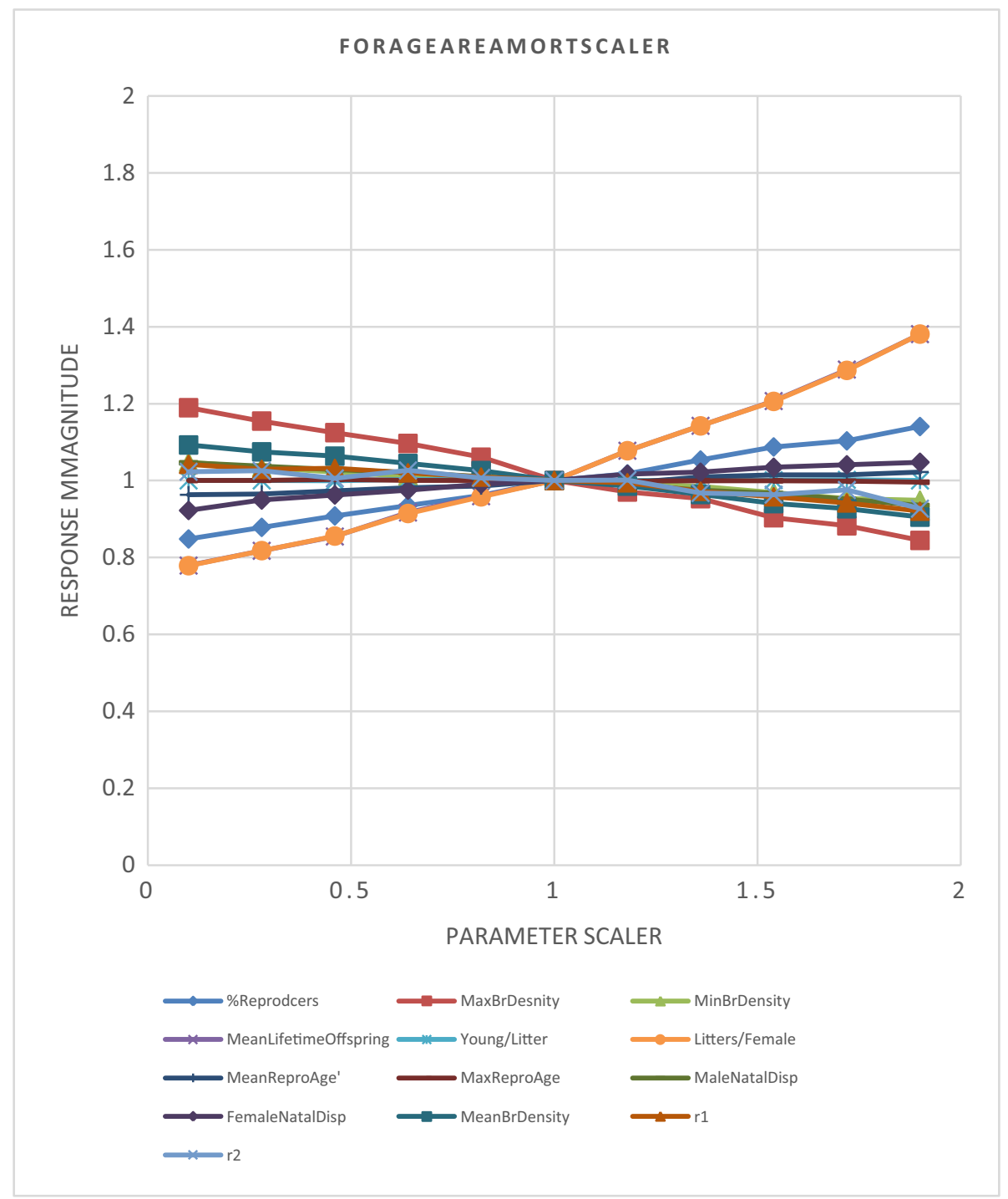

FORAGEAREAMORTSCALER is the constant used to increase bad weather mortality as a function of the proportion of forage available. This multiplier is applied when MINFORAGETEMP is not reached or MAXFORAGERAINFALL is exceeded. 


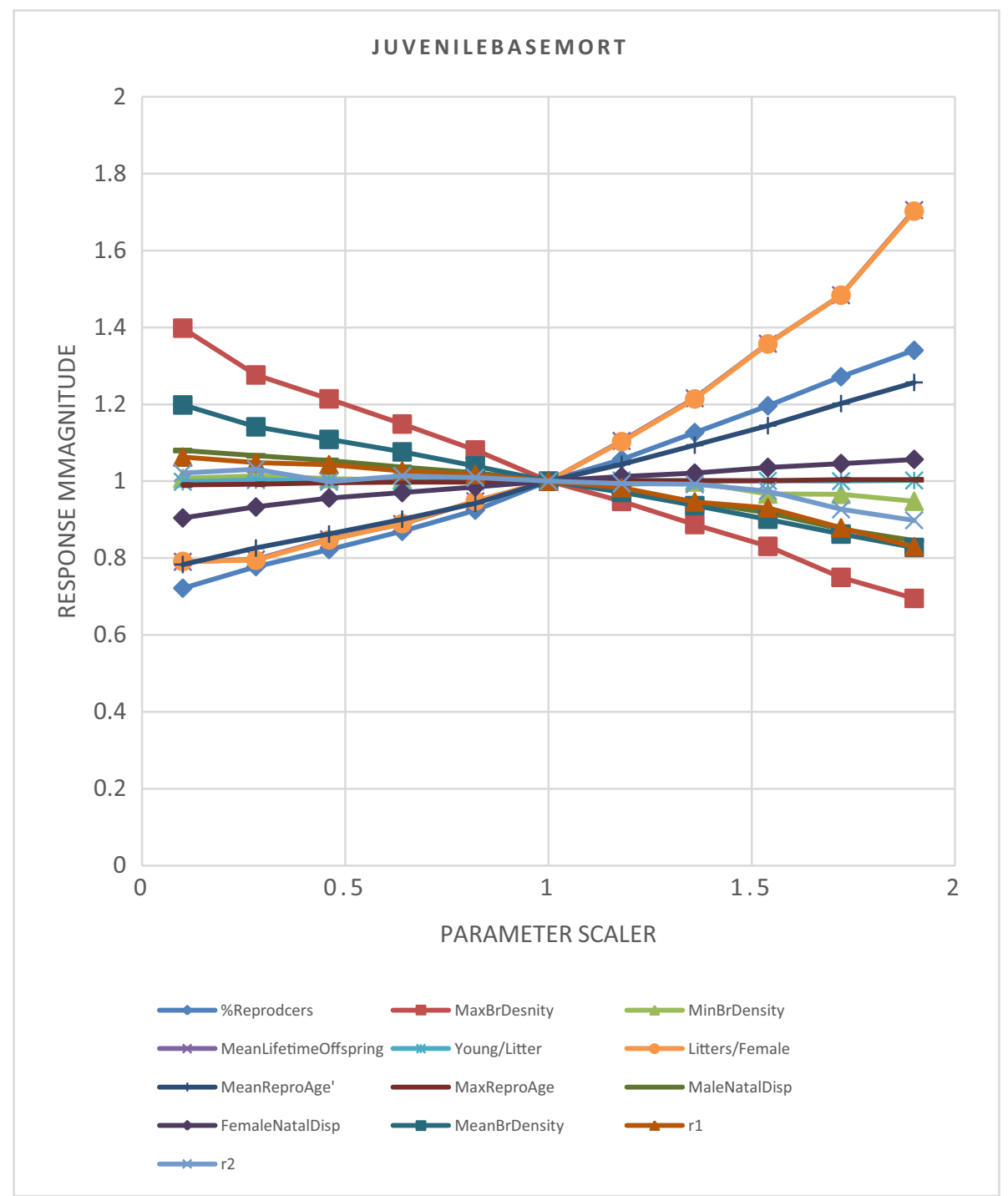

JUVENILEBASEMORT is the daily probability of mortality for the Rabbit_Juvenile stage before any external mortalities or mortality multipliers are applied. 


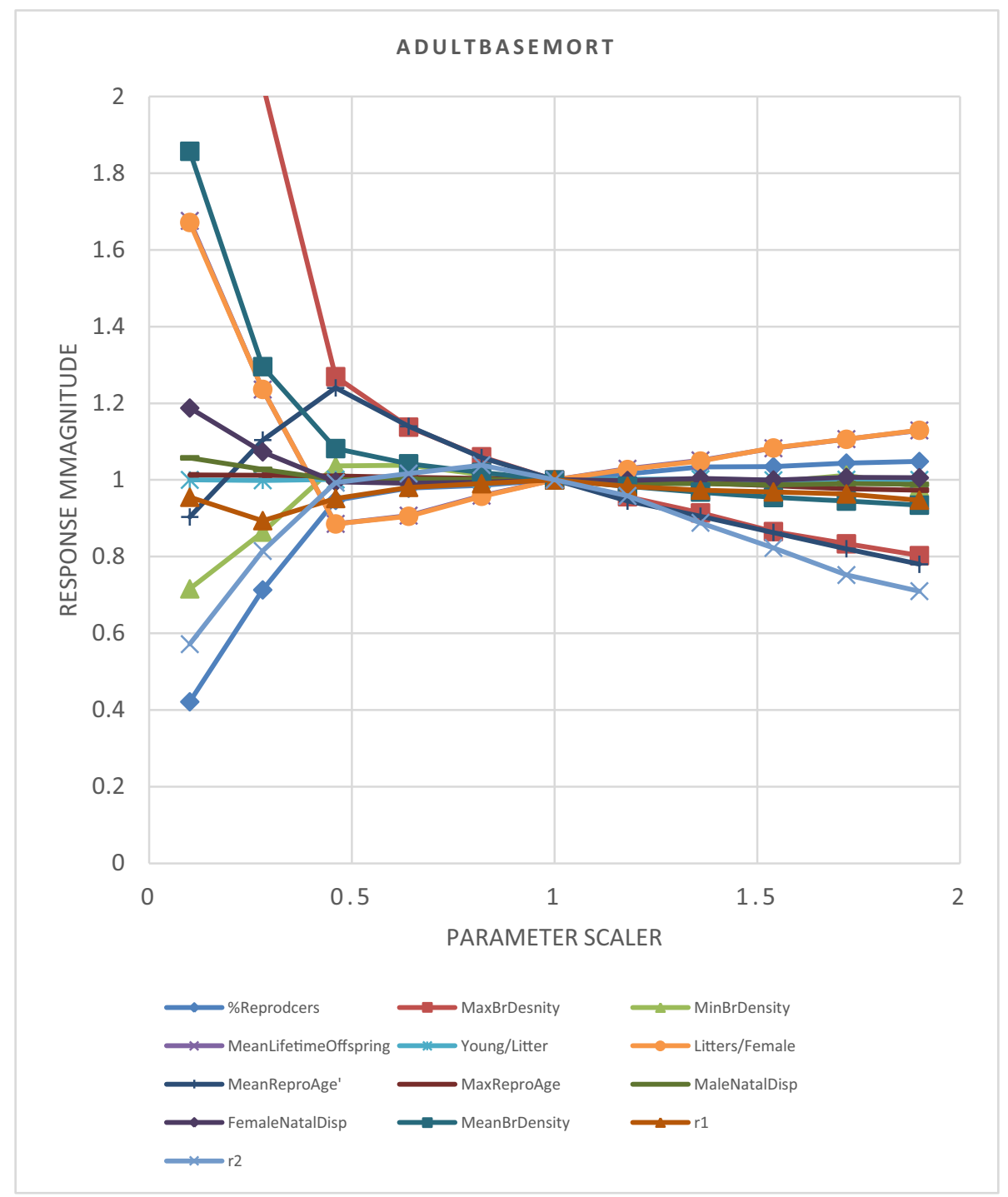

ADULTBASEMORT is the daily probability of mortality for the Rabbit_Adullt stage before any external mortalities or mortality multipliers are applied. 


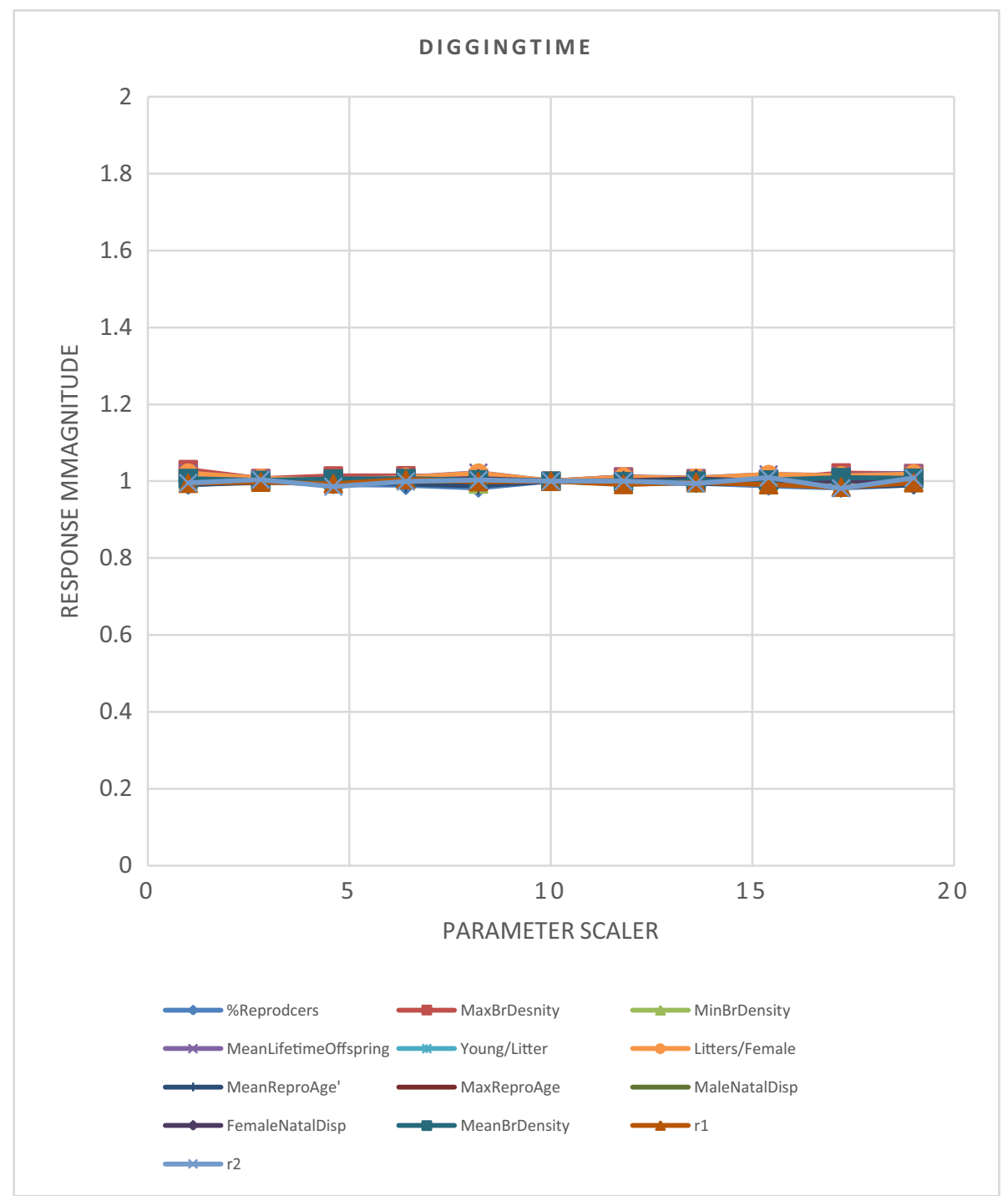

DIGGINGTIME was the time assumed to be taken for a rabbit to dig a new burrow for breeding if one does not exist, and there is room in the warren. 


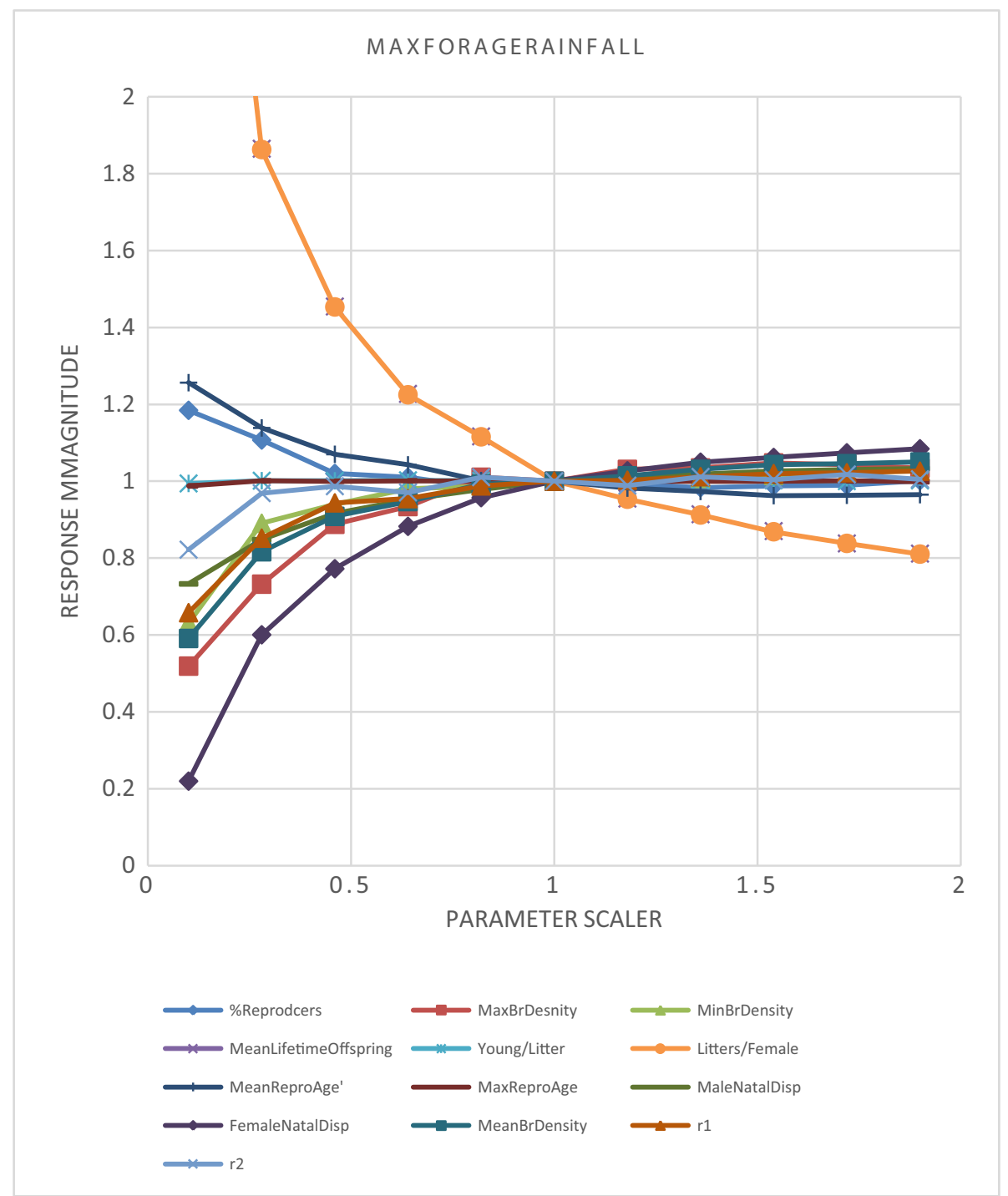

MAXFORAGERAINFALL is the amount of precipitation in mm per day that set as the threshold for efficient foraging to occur. This primarily affects juvenile growth and forage-related mortality. 


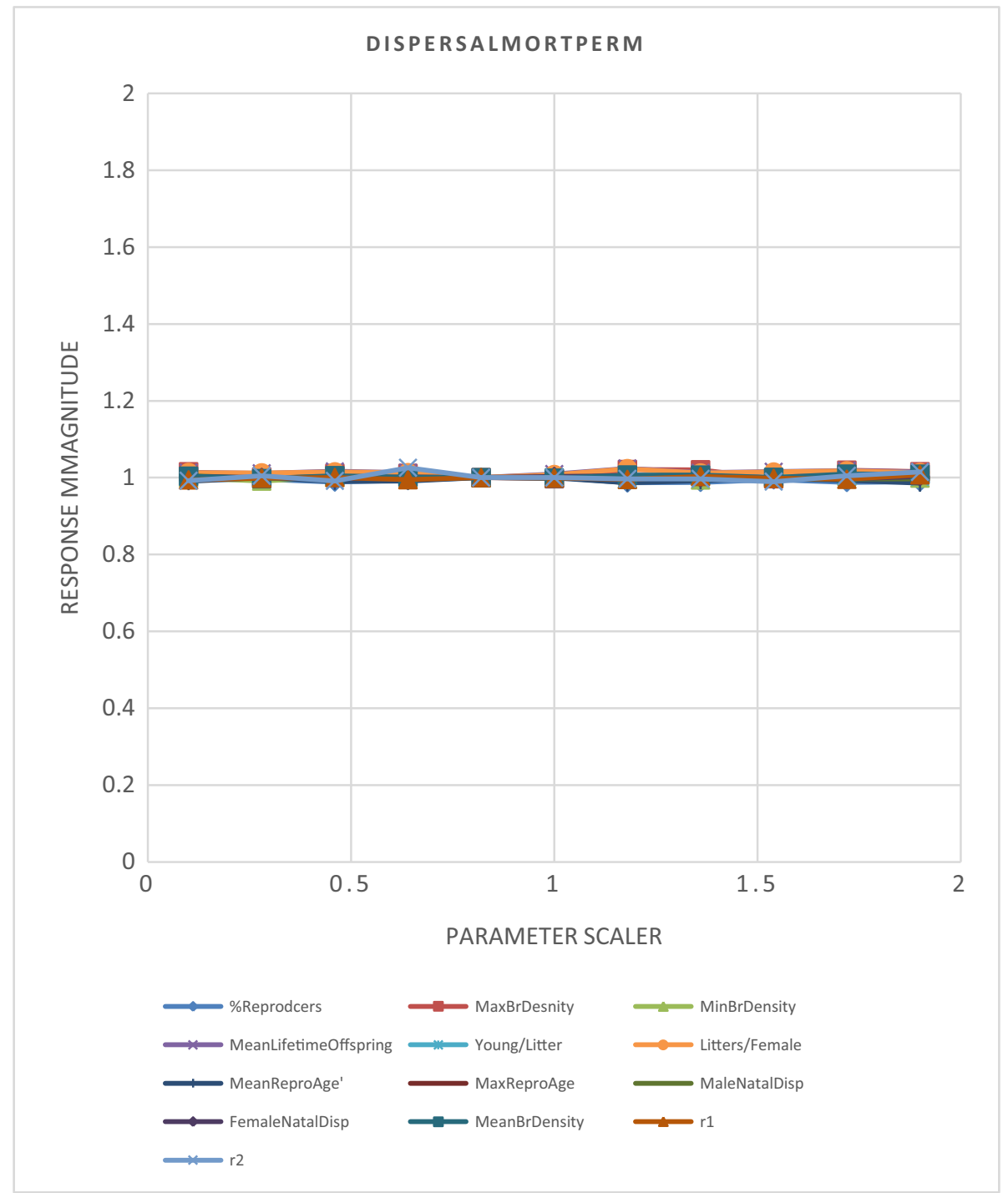

DISPERSALMORTPERM is the extra probability of mortality per metre moved during dispersal for any rabbit.

Open Access This article is distributed under the terms of the Creative Commons Attribution 4.0 International License (http:// creativecommons.org/licenses/by/4.0/), which permits unrestricted use, distribution, and reproduction in any medium, provided you give appropriate credit to the original author(s) and the source, provide a link to the Creative Commons license, and indicate if changes were made.

\section{References}

1. European Council. (2002). SANCO/10329/2002: Guidance document on terrestrial ecotoxicology under council directive 91/414/ EEC. p. 39.
2. EFSA. (2009). Risk assessment for birds and mammals. EFSA Journal. 7(12): p. Article 1438.

3. EFSA. (2016). Scientific committee, Ecological recovery in ERA. EFSA Journal, 14(2), 85.

4. EFSA. (2015). Panel on plant protection products and their residues (PPR), Scientific opinion addressing the state of the science on risk assessment of plant protection products for non-target arthropods. EFSA Journal, 13(2), 3996.

5. Barrio, I. C., Villafuerte, R., \& Tortosa, F. S. (2012). Can cover crops reduce rabbit-induced damages in vineyards in southern Spain? Wildlife Biology, 18(1), 88-96.

6. Barrio, I. C., Villafuerte, R., \& Tortosa, F. S. (2011). Harbouring pests: rabbit warrens in agricultural landscapes. Wildlife Research, 38(8), 756-761. 
7. Nugent, G., et al. (2012). Why $0.02 \%$ ? A review of the basis for current practice in aerial 1080 baiting for rabbits in New Zealand. Wildlife Research, 39(2), 89-103.

8. Wray, S. (2006). A guide to rabbit management. Report CIRIA C645. Construction Industry Reaseach and Information Association (CIRIA).

9. Topping, C. J., Dalby, L., \& Skov, F. (2016). Landscape structure and management alter the outcome of a pesticide ERA: Evaluating impacts of endocrine disruption using the ALMaSS European Brown hare model. Science of the Total Environment, 541, 1477-1488.

10. Topping, C. J., Hoye, T. T., \& Olesen, C. R. (2010). Opening the black box-development, testing and documentation of a mechanistically rich agent-based model. Ecological Modelling, 221(2), 245255.

11. Magliocca, N. R., \& Ellis, E. C. (2013). Using pattern-oriented modeling (POM) to cope with uncertainty in multi-scale agentbased models of land change. Transactions in GIS, 17(6), 883-900.

12. Piou, C., Berger, U., \& Grimm, V. (2009). Proposing an information criterion for individual-based models developed in a patternoriented modelling framework. Ecological Modelling, 220(17), 1957-1967.

13. Wang, M., et al. (2016). Pattern-oriented modelling of plant architecture: A new approach for constructing functional-structural plant models. 2016 Ieee International Conference on FunctionalStructural Plant Growth Modeling, Simulation, Visualization and Applications (Fspma). p. 204-213.

14. Topping, C. J., Odderskaer, P., \& Kahlert, J. (2013). Modelling skylarks (Alauda arvensis) to predict impacts of changes in land management and policy: development and testing of an agentbased model. PLoS One, 8(6), e65803.

15. Topping, C. J., Dalkvist, T., \& Grimm, V. (2012). Post-hoc patternoriented testing and tuning of an existing large model: lessons from the field vole. PLoS One, 7(9), e45872.

16. Grimm, V., \& Railsback, S. F. (2012). Pattern-oriented modelling: a "multiscope" for predictive systems ecology. Philosophical Transactions of the Royal Society of London Series B-Biological Sciences, 367, 298-310.

17. Grimm, V., et al. (2005). Pattern-oriented modeling of agent-based complex systems: lessons from ecology. Science, 310(5750), 987991.

18. Topping, C. J., et al. (2015). Per Aspera ad Astra: through complex population modeling to predictive theory. American Naturalist, 186(5), 669-674.

19. Tarantola, A. (1987). Inverse problem theory: methods for data fitting and model parameter estimation. New York: Elsevier.

20. Kunkele, J., \& von Holst, D. (1996). Natal dispersal in the European wild rabbit. Animal Behaviour, 51, 1047-1059.

21. Rodel, H. G., et al. (2004). Over-winter survival in subadult European rabbits: weather effects, density dependence, and the impact of individual characteristics. Oecologia, 140(4), 566-576.

22. Rodel, H. G., et al. (2005). Timing of breeding and reproductive performance of female European rabbits in response to winter temperature and body mass. Canadian Journal of Zoology-Revue Canadienne De Zoologie, 83(7), 935-942.

23. Rodel, H. G., et al. (2004). Density-dependent reproduction in the European rabbit: a consequence of individual response and agedependent reproductive performance. Oikos, 104(3), 529-539.

24. Rodel, H. G., Monclus, R., \& von Holst, D. (2006). Behavioral styles in European rabbits: social interactions and responses to experimental stressors. Physiology \& Behavior, 89(2), 180-188.

25. von Holst, D., et al. (2002). Social rank, fecundity and lifetime reproductive success in wild European rabbits (Oryctolagus cuniculus). Behavioral Ecology and Sociobiology, 51(3), 245-254.

26. Brambell, F. W. R. (1948). Prenatal mortality in mammals. Biological Reviews of the Cambridge Philosophical Society, 23(4), 370-407.
27. Boyd, I. L., \& Myhill, D. G. (1987). Seasonal-changes in condition, reproduction and fecundity in the wild European rabbit (Oryctolagus-Cuniculus). Journal of Zoology, 212, 223-233.

28. McIlwaine, C. P. (1962). Reproduction and body weights of the wild rabbit Oryctolagus cuniculus (L.) in Hawke's Bay, New Zealand. New Zealand Journal of Science, 5, 325-341.

29. Parer, I., \& Libke, J. A. (1991). Biology of the wild rabbit, Oryctolagus-cuniculus (L), in the Southern Tablelands of NewSouth-Wales. Wildlife Research, 18(3), 327-341.

30. Brambell, F. W. R. (1942). Intra-uterine mortality of the wild rabbit, Oryctolagus cuniculus (L). Proceedings of the Royal Society BBiological Sciences, 130(861), 462-479.

31. Brambell, F. W. R. (1944). The reproduction of the wild rabbit Oryctolagus cuniculus (L). Proceedings of the Zoological Society of London, 114, 1-45.

32. Mykytowycz, R., \& Fullagar, P. J. (1973). Effect of social environment on reproduction in the rabbit, Oryctolagus cuniculus (L.) Journal of reproduction and fertility Supplement, 19, 503-522.

33. Boyd, I. L. (1985). Investment in Growth by pregnant wild rabbits in relation to litter size and sex of the offspring. Journal of Animal Ecology, 54(1), 137-147.

34. Trout, R. C., \& Smith, G. C. (1995). The reproductive productivity of the wild rabbit (Oryctolagus-cuniculus) in southern England on sites with different soils. Journal of Zoology, 237, 411-422.

35. Trout, R. C., \& Smith, G. C. (1998). Long-term study of litter size in relation to population density in rabbits (Oryctolagus cuniculus) in Lincolnshire. England. Journal of Zoology, 246, 347-350.

36. Wallagedrees, J. M., \& Michielsen, N. C. (1989). The influence of food supply on the population dynamics of rabbits, Oryctolagus cuniculus (L), in a Dutch dune area. Zeitschrift Fur Saugetierkunde-International Journal of Mammalian Biology, 54(5), 304-323.

37. Thompson, H. V., \& King, C. M. (1994). The European rabbit. The history and biology of a successful colonizer (p. 245). Oxford: Oxford University Press.

38. Meriggi, A. (2001). Il coniglio selvatico. In C. Prigioni, M. Cantini, \& A. Zilio (Eds.), Atlante dei Mammiferi della Lombardia. Regione Lombardia. p. 130-133.

39. Siracusa, A. M., \& Petralia, E. (2013). Trend of a population of wild rabbit Oryctolagus cuniculus (Linnaeus, 1758) in relation to Domestic Sheep Ovis aries aries (Linnaeus, 1758) grazing within a small insular protected area. Biodiversity Journal, 4(4), 557-564.

40. Barrio, I. C., Bueno, C. G., \& Tortosa, F. S. (2009). Improving predictions of the location and use of warrens in sensitive rabbit populations. Animal Conservation, 12(5), 426-433.

41. Gea-Izquierdo, G., Munoz-Igualada, J., \& San Miguel-Ayanz, A. (2005). Rabbit warren distribution in relation to pasture communities in Mediterranean habitats: consequences for management of rabbit populations. Wildlife Research, 32(8), 723-731.

42. Topping, C. J., Dalby, L., \& Skov, F. (2016). Landscape structure and management alter the outcome of a pesticide ERA: evaluating impacts of endocrine disruption using the ALMaSS European Brown hare model. Science Total Environment, 541, 1477-1488.

43. Smith, G. C., Pugh, B., \& Trout, R. C. (1995). Age and sex bias in samples of wild rabbits, Oryctolagus cuniculus, from wild populations in southern England. New Zealand Journal of Zoology, 22(2), 115-121.

44. Fernandez, C., \& Ceballos, O. (1990). Uneven sex-ratio of wild rabbits taken by golden eagles. Ornis Scandinavica, 21(3), 236-238.

45. Cooke, B. D. (2012). Rabbits: manageable environmental pests or participants in new Australian ecosystems? Wildlife Research, 39(4), 279-289.

46. Bell, D. J., \& Webb, N. J. (1991). Effects of climate on reproduction in the European wild rabbit (Oryctolagus-cuniculus). Journal of Zoology, 224, 639-648. 
47. Hoye, T. T., Skov, F., \& Topping, C. J. (2012). Interpreting outputs of agent-based models using abundance-occupancy relationships. Ecological Indicators, 20, 221-227.

48. Norbury, G., \& Jones, C. (2015). Pests controlling pests: does predator control lead to greater European rabbit abundance in Australasia? Mammal Review, 45(2), 79-87.

49. Spromberg, J. A., John, B. M., \& Landis, W. G. (1998). Metapopulation dynamics: indirect effects and multiple distinct outcomes in ecological risk assessment. Environmental Toxicology and Chemistry, 17(8), 1640-1649.

50. Brock, T. C. M., et al. (2010). Macroinvertebrate responses to insecticide application between sprayed and adjacent non-sprayed ditch sections of different sizes. Environmental Toxicology and Chemistry, 29, 1994-2008.

51. Topping, C. J., et al. (2015). Towards a landscape scale management of pesticides: ERA using changes in modelled occupancy and abundance to assess long-term population impacts of pesticides. Science of the Total Environment, 537, 159-169.

52. Smith, G. C., Prickett, A. J., \& Cowan, D. P. (2007). Costs and benefits of rabbit control options at the local level. International Journal of Pest Management, 53(4), 317-321.

53. Smith, R. K., Jennings, N. V., \& Harris, S. (2005). A quantitative analysis of the abundance and demography of European hares
Lepus europaeus in relation to habitat type, intensity of agriculture and climate. Mammal Review, 35(1), 1-24.

54. Smith, A.T., \& Johnston, C.H. (2008). Lepus europaeus. IUCN Red List of Threatened Species IUCN. 603 2011(2).

55. Smith, R. K., et al. (2004). Conservation of European hares Lepus europaeus in Britain: is increasing habitat heterogeneity in farmland the answer? Journal of Applied Ecology, 41(6), 1092-1102.

56. van der Vaart, E., et al. (2015). Calibration and evaluation of individual-based models using approximate Bayesian computation. Ecological Modelling, 312, 182-190.

57. Campolongo, F., Cariboni, J., \& Saltelli, A. (2007). An effective screening design for sensitivity analysis of large models. Environmental Modelling \& Software, 22(10), 1509-1518.

58. Haythorne, S., \& Skabar, A. (2013). An improved pattern-guided evolution approach for the development of adaptive individualbased ecological models. Ecological Modelling, 252, 72-82.

59. Kubicek, A., et al. (2015). Context-oriented model validation of individual-based models in ecology: a hierarchically structured approach to validate qualitative, compositional and quantitative characteristics. Ecological Complexity, 22, 178-191.

60. EFSA Scientific Committee. (2016). Recovery in environmental risk assessments at EFSA. EFSA Journal, 14(2), 4313 [85pp.] 